\title{
Energy Dissipation Capability and Impact Response of Carbon Nanotube Buckypaper: A Coarse-Grained Molecular Dynamics Study
}

\author{
Heng Chen ${ }^{a b}$, Liuyang Zhang ${ }^{b}$, Jinbao Chen ${ }^{a}$, Matthew Becton ${ }^{b}$, \\ Xianqiao $\mathrm{Wang}^{b}$, and Hong $\mathrm{Nie}^{a}$, \\ ${ }^{a}$ College of Aerospace Engineering, Nanjing University of Aeronautics and Astronautics, \\ Nanjing, Jiangsu 210016 China \\ ${ }^{b}$ College of Engineering, University of Georgia, Athens, GA 30602 USA
}

\begin{abstract}
Carbon nanotube (CNT) buckypaper, a randomly non-woven fibrous film structure, has enjoyed its popularity in the sensor, actuator, filtration, and distillation devices owing to its exceptional mechanical and electrical properties. However, there is no report aimed at unraveling the fundamental mechanism of its energy-absorption capability under high-velocity impact despite its extraordinary frequency- and temperature-invariant viscoelastic properties. To bridge this gap, here coarse-grained molecular dynamics simulations are implemented to investigate effects of the external impact energy, the density of the buckypaper, and the length of individual CNTs on energy dissipation capability and dynamic response of the buckypaper under high-velocity impacts. Simulation results indicate that within its deformation limit the buckypaper possesses extremely high kinetic energy dissipation efficiency. The critical impact energy related to the deformation limit of the buckypaper tightly depends on the impact velocity since the same impact energy with a larger impact velocity yields less compression. The energy dissipation capability and impact response of the buckypaper are demonstrated to be independent of the length of individual SWCNTs. Overall, owing to the remarkable energy dissipation capability and flexibility of the buckypaper, it can be regarded as a promising candidate for energy dissipation.
\end{abstract}




\section{Introduction}

Energy dissipation materials and structures play a vital role in the safety of structures and humans subjected to a crash event [1]. Traditionally metals have been widely used for the energy absorption devices, relying on its structural failure and plastic deformation to mitigate impact energy [2, 3]. Cellular forms including foams, honeycombs, and sandwich structures also perform excellently in mitigating dynamic loadings due to bulking and collapse mechanisms [411]. In addition, internal damping caused by the unique internal structure of composites exhibits favorable performance for the control of vibration due to impact $[12,13]$.

In order to meet the ever-increasing high-demand on lightweight, small volume, and high energy dissipation efficiency systems, nanostructured materials have begun attracting researchers' interest for their excellent mechanical properties such as enhanced strength-to-weight, surfaceto-volume, and stiffness-to-weight ratios. For example, nanocomposites with reinforcement fibers or particles possess significantly enhanced property such as volume fraction compared with the corresponding composites with macro-scale fibers or particles $[14,15]$. Especially, welldesigned polymer-based nanomaterials have superior impact behavior and much higher energy dissipation density than conventional materials due to their high impact toughness [16]. Nanoporous materials also have been proven to possess fantastic energy dissipation capabilities owing to their small pore size, high porosity, and high network strength [17-19]. Han at el. found that nanoporous silica had energy dissipation density on the order of $300 \mathrm{~J} / \mathrm{g}$, much larger than that of conventional materials, and the dissipation mechanism is the non-uniform collapse of nanopores and subsequent yielding via permanent plastic deformations [20]. Nanoporous systems consisting of non-wetting liquid and nanoporous particles show energy dissipation density as high as $\sim 18 \mathrm{~J} / \mathrm{cm}^{3}[21-25]$. 
Fullerenes such as carbon nanotubes (CNTs) and buckyballs have been demonstrated to have unprecedented mechanical properties and energy absorption capability owing to significantly high strength and stiffness, large surface area, and light weight [26-33]. For example, CNTs can exhibit a Young's modulus of over $1 \mathrm{TPa}$ and tensile strength above $100 \mathrm{GPa}[34,35]$. Xu at el. [36-38] found that buckyballs with larger size tend to yield non-recoverable deformation which assists energy dissipation thanks to the unique deformation characteristic dependent on the buckyball size under dynamic loadings. An energy absorption system of buckyballs confined by a CNT was put forward and found to have remarkable energy absorption density of $2000 \mathrm{~J} / \mathrm{g}$ [39]. CNT-based nanocomposites, such as aligned CNT/epoxy nanocomposites, have improved properties beneficial for energy dissipation by increasing CNT volume fraction and CNT aspect ratio [40-43]. Assembled CNTs with controlled orientation and configurations, such as CNT foams and three-dimensional sponge-array architectures, have exhibited unprecedented mechanical properties and energy dissipation capability [44-46].

Recently, both experimental and computational results reveal that the long-ranged van der Waals interactions between CNTs enable them to aggregate and thus form CNT networks [47-49] called 'buckypaper'. Buckypaper has been verified to show intriguing mechanical and thermal properties attributed to the excellent properties of individual CNTs, enabling it to be one of the most promising nanomaterials [50-53]. Experimental results indicate that the Young's modulus of buckypapers with different densities and CNT diameters can be tuned from $0.2 \mathrm{GPa}$ to 3.1 GPa [54, 55]. Li [56] found that entanglement and bundling mechanisms play an important role in the mechanical properties of buckypapers. Xie [57] found that the microstructures of buckypapers undergo a remarkable evolution under mechanical loadings. Both experimental and computational results reveal that buckypapers exhibit frequency- and temperature-invariant 
viscoelastic properties from -196 to $1000^{\circ} \mathrm{C}[58,59]$ and a zipping-unzipping mechanism is observed under cyclic loadings [60]. Buckypapers usually feature low densities of $0.05-$ $0.4 \mathrm{~g} / \mathrm{cm}^{3}$ and high porosities of $0.8-0.9[61]$, and the pore size of SWCNT (diameter $0.8-1.2 \mathrm{~nm}$ with length $100-1000 \mathrm{~nm}$ ) buckypaper was reported around $10 \mathrm{~nm}$ [62]. All these results indicate that buckypapers may possess favorable properties for energy dissipation, but few studies have focused on this application. Therefore, this paper will investigate the impact behavior and energy dissipation capability of buckypapers by utilizing coarse-grained molecular dynamics simulations, in terms of effects of impact energy, density of buckypapers, and length of individual SWCNTs as well as the evolution of the deformation. The results enable a holistic picture of impact performance of buckypapers and highlight a promising candidate for energy dissipation under extreme conditions.

\section{Model and computational Methods}

\subsection{Coarse-grained model for CNT}

As full atomistic molecular dynamics simulations are computationally expensive for long interacted CNT networks, in order to enhance computational efficiency it calls for mesoscale modeling $[60,63]$. Here a coarse-grained molecular dynamics model is applied to effectively express the mechanical properties of CNTs [55] over relatively large length scales. Our coarsegrained molecular dynamics (CGMD) simulations are performed on the open source platform LAMMPS [64]. In CGMD model of CNT network, each CNT is simplified as a multi-bead chain. For a certain chain, stretching and bending properties are reflected through bonds between two adjacent beads and angles formed by three successive beads, respectively. Long-ranged van der Waals (vdW) interaction between pairs of beads accounts for the inter-tube interaction. CNTs' twisting behavior, usually neglected in current papers about computational model of 
buckypaper, can make marginal effect on the absolute value of our results. And the corrugation effect resulted from the nature of the bead-spring model when CNTs slide against each other is regardless in this paper. Since this CG model and its relevant force field regardless of the corrugation effect have been proved by many researchers to successfully describe different mechanical properties of the buckypaper, such as the entanglement and bundling phenomenon, the structural and mechanical properties, the visco-elastic properties of the buckypaper and so on $[56,58,60,65,66]$, the corrugated effect is assumed to be marginal. Thus, the total energy of the coarse-grained system is given by:

$$
E_{\text {tot }}=E_{\text {bond }}+E_{\text {angle }}+E_{v d W}
$$

where $E_{\text {bond }}=\sum_{\text {bonds }} \frac{1}{2} k_{b}\left(r-r_{0}\right)^{2}$ denotes the inter-chain stretching energy along the axial direction, $k_{b}$ is the stretching constant, $r$ is the current bond length and $r_{0}$ is the equilibrium bond length. $E_{\text {angle }}=\sum_{\text {angles }} \frac{1}{2} k_{a}\left(\theta-\theta_{0}\right)^{2}$ represents the bending energy, where $k_{a}$ is the bending constant, and $\theta$ and $\theta_{0}$ express the current angle and the equilibrium angle respectively. $E_{v d W}=\sum_{\text {pairs }} 4 \varepsilon\left[(\sigma / r)^{12}-(\sigma / r)^{6}\right]$, a pairwise 12-6 Lennard-Jones potential, depicts the van der Waals interaction with $\varepsilon$ describing the depth of the potential well and $\sigma$ describing the equilibrium distance where the inter-bead potential is zero. These six parameters $k_{b}, r_{0}, k_{a}, \theta_{0}, \varepsilon$ and $\sigma$ can be derived from a full atomistic computational experiments using the Tersoff potential or ReaxFF potential $[67,68]$. According to Buehler and Cranford's research $[69,70], r_{0}, \theta_{0}$, and $\sigma$ can be determined by equilibrium conditions while $k_{b}, k_{a}$ and $\varepsilon$ can be obtained by energy conservation calculations. In detail, uniaxial tension tests of a single CNT can be applied to describe the stretching behavior of CNTs and calculate the Young's modulus $E$ and then the equivalent $k_{b}$ equal to $E A / r_{0}$ ( $A$ is the cross-section area of the CNT) can be obtained. Bending 
tests can determine the bending behavior and bending stiffness $E I$ ( $I$ is the bending moment of inertia) of the CNT and thus $k_{a}=3 E I / 2 b_{0}$ can be gained. Simulations of an atomistic assembly of two CNTs can calculate the equilibrium distance $D$ between them and the adhesive strength $\beta$, and thereby $\varepsilon$ and $\sigma$ can be acquired via $\varepsilon=\beta r_{0}$ and $\sigma=D / \sqrt[6]{2}$ respectively.

In this work, a buckypaper is modeled as if formed by $(5,5)$ SWCNTs and the potential parameters of the coarse-grained model are depicted in Table 1. The parameters obtained from the full atomistic simulation have been validated by tensile test, self-folding of CNTs, selfassembly process, and behavior of CNT bundles [55, 69, 70]. The tensile stress-strain relationship reported by Buehler is found to agree well with the experimental results reported by Peng [35]. The cutoff distance of the long-ranged van der Waals interactions is set to be $9.35 \mathrm{~nm}$. Based on the mass per unit length of SWCNT and the equilibrium bead distance, the mass of each bead is given by $1,953.23 \mathrm{~g} / \mathrm{mol}$. For all the simulations results, the total length of all the SWCNTs in the buckypaper is fixed to be $51,200 \mathrm{~nm}$. In terms of effects of impact energy and density, the buckypaper consists of 512 SWCNTs with individual length of $100 \mathrm{~nm}$. In order to investigate the effects of SWCNT length, SWCNTs with different lengths $100 \mathrm{~nm}$, $149 \mathrm{~nm}, 237 \mathrm{~nm}, 410 \mathrm{~nm}$, and $800 \mathrm{~nm}$ are used to construct five types of buckypapers respectively, keeping the density and total SWCNTs length unchanged.

\subsection{Configurations of buckypaper}

In order to ensure the randomness and isotropy, the random walk method is adopted to generate the buckypaper. Toward this end, a series of points with the number denoted as $n_{p}$ are uniformly distributed in an orthogonal cell by setting a certain number of seeds $\left(n_{x}, n_{y}, n_{z}\right)$ along the three axes respectively. Taking each point as the initial bead, a SWCNT grows to the next bead via a 
random bond vector with the length as the equilibrium bead distance $10 \AA$. Then an overlap check is conducted to avoid the overlap of beads. That is, if the distance between this bead and its nearest bead is less than a specific distance ( $2 \AA)$, the new generated position is considered to be occupied by another bead and therefore this SWCNT will return one step to the last position and then proceed via a new random vector. This check process is repeated until the new position is not occupied and then the new bead is generated. Once the distance of the random walk equals the SWCNT length required for investigation, the walk stops and the current bead is the terminal bead of the SWCNT. It can be seen that the number of points $\left(n_{p}=n_{x} \times n_{y} \times n_{z}\right)$ and the walk steps determine the number of SWCNTs and the length of each SWCNT, respectively. In this work, all the SWCNTs in a single cell have the same length. In terms of density effects, the density of buckypaper is changed by tuning the distance between the adjacent seeds along each axis and thus tuning the density of distributed points. After the structure is equilibrated, the density varies from $40.67 \mathrm{~kg} / \mathrm{m}^{3}$ to $107.60 \mathrm{~kg} / \mathrm{m}^{3}$ which closely matches the value obtained from experiments [59] and simulations [58].

Afterwards the initial buckypaper (Figure 1(a)) is relaxed based on an isothermal-isobaric (NPT) ensemble to perform the free energy minimization. During the equilibrium process (Figure 1(a) Figure 1(b)), the temperature and the pressure are driven to a constant $300 \mathrm{~K}$ and $1 \mathrm{~atm}$ respectively. The SWCNTs appear to be entangled and aggregated due to the van der Waals interactions. Two dynamic standard 12-6 L-J walls are added outside both the upper and lower surfaces and always keep $10 \AA$ away from the buckypaper, for the convenience of adding two rigid plates needed for the following impact simulations. During the relaxation, certain SWCNTs begin to attach each other and zip together (Figure 1(d)-(e)), which is also observed in references $[58,60]$. The total energy of the buckypaper drops rapidly in the first $3 \mathrm{~ns}$ and then achieves an 
equilibrium value slowly in the subsequent $4 \mathrm{~ns}$. For validation of the buckypaper model, MD simulations for static compression and shear tests are performed and it is found that both the stress-strain curve and the elastic modulus (Figure S3-Figure S5) agree well with that provided in Li et al. [56]. The Young's modulus obtained in our simulations is around $0.01-0.1 \mathrm{GPa}$, slightly lower than that reported by experiments $[72,73]$ around $0.2-12 \mathrm{GPa}$ because of the lower density of buckypaper. Hence, the model of buckypaper used in this paper can be considered to be reasonable.

As mentioned before, after the fully relaxation of buckypaper the L-J walls are removed and two rigid plates with the same length and width are added to the system. As is depicted in Figure 1(c), one plate (impactor) is right above the buckypaper and $20 \AA$ from its upper surface while the other one (receiver) is fixed under the buckypaper and $20 \AA \AA$ from its lower surface. Impact energy $\left(E_{\text {impact }}\right)$ is achieved by endowing the whole impactor with an initial velocity $\left(v_{0}\right)$ along the $z$ direction. A microcanonical ensemble (NVE) is employed so that the total energy of the system remains unchanged. Periodic boundary conditions are remained in both $x$ and $y$ dimensions while aperiodic conditions are applied to the $z$ dimension. Timestep for all the simulations is $10 \mathrm{fs}$.

\section{Results and Discussions}

\subsection{Effects of impact energy}

In order to understand the effect of impact energy on the energy dissipation capability and impact response of the buckypaper, a series of impact energies ranging from $E_{0}$ to $10 E_{0}\left(E_{0}=\right.$ $1.5 \mathrm{fJ})$ are applied to impact the buckypaper. The impact energy can be varied by either tuning the initial velocity or the mass of the impactor. For better investigation of their effects, two 
methods, noted as FM (fixing mass and changing velocity) and FV (fixing velocity and changing mass), respectively, are both adopted. For FV cases, the initial velocity of the impactor is fixed as $200 \mathrm{~m} / \mathrm{s}$ while the mass $(\mathrm{m})$ varies from $7.5 \times 10^{-17} \mathrm{~g}$ to $7.5 \times 10^{-16} \mathrm{~g}$. For FM cases, the mass of the impactor is fixed as $3.7 \times 10^{-17} \mathrm{~g}$ while the initial velocity varies from $282.8 \mathrm{~m} / \mathrm{s}$ to $894.4 \mathrm{~m} / \mathrm{s}$. This velocity regime is conventionally considered as high impact speed domain, mainly aiming at the ballistic impact related problem. By observing the animation of the impact process in the visualization software OVITO [74], it is found that as the impactor goes downwards the SWCNTs in the upper irregular region start to be compressed, and thus a narrow densified region appears on top of the buckypaper system as shown in Figure 2(b). Due to the continuation of the compression and the propagation of the stress wave induced by the impactor, it causes the extension of the densification region in the upper part of the buckypaper, as shown in Figure 2(c). It is also noticed that SWCNTs in the lower part of the buckypaper are not affected by the loading at the early stage of impact. Afterwards the densification region moves downwards and transfers the straining to the rest of the buckypaper structure gradually, thus more SWCNTs get involved into densification. Finally those SWCNTs in the lowest part are exposed to impact and a flat surface is formed as a result of the fixed receiver. As is demonstrated in Figure 2(d), the region near the upper and lower surfaces is denser than the middle part of the buckypaper. It is noted that in the densifying procedure SWCNTs are detached and attached cyclically through the unzipping-zipping mechanism at these entanglement sites. As is depicted in Figure 3, the two partially attached (violet) SWCNTs in Figure 3(a) detach in Figure 3(b) and then combine with another (violet) CNT forming a new attachment of longer length in Figure 3(c). Then they detach in Figure 3(d) but attach with each other again rapidly in Figure 3(e); this process is repeated frequently during the rest of the impact process. The reason 
is probably that the nonlinear dynamic impact behavior of buckypaper results in the fluctuation of individual SWCNTs. This detachment-attachment process enables energy dissipation, because energy is consumed when SWCNTs overcome the van der Waals interactions during the detachment process. When the impactor velocity is damped to zero, the impactor has the largest displacement and the buckypaper goes to the deformation extreme. Afterwards the impactor rebounds slightly, seen in Figure 2(e) and (f). Herein, we define the rebound distance of the buckypaper to be the displacement of the upper surface of the buckypaper from the impactor, reaching the maximum displacement upon detaching from the buckypaper. It is noticed that during the impact process individual CNTs hardly have very large deformation and thus it's reasonable to make an elastic assumption of individual CNTs while the collective behaviors from many individual CNTs can be plastic deformation. During the impact process, the kinetic energy of the impactor is transferred to potential energy (strain energy) and thermal energy of the buckypaper resulting in a temperature increase of the buckypaper. Potential energy includes vdW pairwise energy, bond energy, and angle energy. As shown in Figure S1, the vdW pairwise energy decreases during the impact process, resulting in a stronger vdW interactions than the initial one. However, both bond energy and angle energy increase during the impact process due to the compression deformation.

To better understand the deformation mechanism of the buckypaper in the impact process, the upper contact force $\left(F_{i}\right)$ between the impactor and the buckypaper and the lower contact force $\left(F_{r}\right)$ between the buckypaper and the receiver are monitored. According to the comparison between them depicted in Figure 4, it is found that the upper contact force increases quickly at the early stage of compression since more and more SWCNTs are involved in resisting the compression, and then the upper contact force reaches a local peak with the buckypaper morphology in Figure 
2(b). During this procedure the buckypaper remains relaxed and unaffected, except for a small portion on the top. As the impactor goes down, the densification region forms to resist further compression and a stress wave is introduced to its neighboring part under the impactor. The densification region is not being compressed to its limit and the upper contact force decreases until the bottom part of the buckypaper receives the compression signal (seen in Figure 2(c)). Supported by the receiver, the lower surface of the buckypaper starts flat and the interaction between the buckypaper and the receiver becomes apparent. As the region near the lower surface of the buckypaper becomes more and more densified, the lower contact force increases and then surpasses the upper contact force. It has to be noted that the first peak value of the lower contact force is greatly less than that of the upper contact force, which is because that the power of the stress wave is attenuated considerably during the propagation process. When the impactor reaches its maximum displacement with apparent pores still left in the buckypaper from the side view, the buckypaper is regarded to still have sufficient room for deformation, as illustrated in the snapshot of Figure 5(D). Both contact forces fluctuate slightly until the rebound of the impactor, such as the case of FV at $3 E_{0}$ and FM at both $3 E_{0}$ and $6 E_{0}$ in Figure 4 . Otherwise if the buckypaper is so tight that no pore is observed in the buckypaper from the side view, the buckypaper is regarded to reach its effective deformation limitation as depicted in the snapshot of Figure 5(C), resulting in a significant increase of the compression stiffness. As the buckypaper becomes denser, the compression stiffness gets larger. Therefore, as the compression continues both contact forces increase and tend to reach the peaks at the maximum compression followed by the rebound of the impactor. Accompanied with the rebound of the impactor both contact forces in all cases keep decreasing and become zero upon the detachment of the impactor from the buckypaper. By comparison of the contact forces in FV and FM cases under the same impact 
energy, at the early stage the upper force in FM case grows more rapidly to a larger peak than that in FV case, because the larger initial velocity of the impactor in FM case results in a shorter propagation distance of the straining and thus a larger resistant stiffness. As follows, this force in FM case decreases more rapidly to a smaller value than that in FV case. In addition, the amplitude of the lower contact force in FM case is lower than that in FV case since more energy is dissipated before the straining arrives at the receiver.

Based on the work-kinetic energy relationship, the work done by the impactor equals to the transferred kinetic energy of the impactor and can be represented by the area surrounded by the upper contact force curve and the horizontal axis in Figure 5. Comparing Figure 4 with Figure 5, we notice that at the same displacement of the impactor, more impact energy is attenuated in the FM case than that in the FV case with the same impact energy, resulting in a shorter compression distance in the FM cases than in the FV cases. Also in the FM cases, with the increment of the impact energy from $3 E_{0}$ to $8 E_{0}$, more energy dissipation arises at the same displacement of the impactor, whereas in the FV cases the energy dissipation has no significant difference. The reason is that the impact velocity of the impactor plays a dominant role in the deformation evolution of the buckypaper. For a larger velocity, the impactor takes a shorter time to reach the same displacement and the propagation distance of the straining is shorter, leading to a smaller yet more compact densification region. For example, the snapshots (A) and (B) in Figure 5 show the morphologies of the buckypaper under the impact energy $8 E_{0}$ for the FV and FM cases respectively when the displacement of the impactor is $60 \mathrm{~nm}$. It can be seen that the region near the lower surface of the buckypaper in (A) has been compressed while that of (B) is not. Additionally, the top region (B) is more densified than that in (A). Snapshots (C) and (D) represent their morphologies at the maximum compression distance. The buckypaper in (C) has 
completely deformed, in response to the rapid increment of the contact force as it approaches the maximum compression. Yet, the buckypaper in (D) has superior deformation ability, and thus at this stage the force remains small.

Therefore, as depicted in Figure 6, for a lower impact energy that enables the buckypaper to have adequate deformation capability, the maximum upper contact force appears at the early stage of the impact and increases slowly along with the impact energy. In addition, the upper contact force for the FM case is higher than that for the FV case. For a large impact energy that causes the buckypaper to compress beyond its deformation limit, the maximum force occurs at the maximum compression. With the growth of the impact energy, the force increases rapidly because of the abrupt increment of the compression stiffness, and after a certain impact energy it exceeds that of the FM case which increases steadily. Figure 6 also shows that both contact forces in the FV case are very close to each other while the lower contact force is noticeably smaller than the upper contact force in the FM case. The difference for both forces in the FM cases becomes pronounced with the growth of the impact energy. The results indicate that a larger impact velocity means that more energy is attenuated before being transmitted to the receiver.

The relationship between the maximum displacement of the impactor and the impact energy is described in Figure 7. With the increment of the impact energy, the rebound distance of the buckypaper stays approximately constant in both FM and FV cases, and is always in the range of $15 \mathrm{~nm}$ to $20 \mathrm{~nm}$. The maximum displacement of the impactor varies widely with different impact energies. For FV cases, it increases from $43 \mathrm{~nm}$ to $117 \mathrm{~nm}$ while the impact energy rises from $E_{0}$ to $6 E_{0}$. Then it increases steadily because of the deformation limit of the buckypaper. For FM cases, with the impact energy varying from $E_{0}$ to $10 E_{0}$, it keeps increasing from $33 \mathrm{~nm}$ 
to $101 \mathrm{~nm}$. The pattern of the curve indicates that it continues to increase if larger impact energy is applied due to the buckypaper's superior deformation capability. With the growth of the impact energy the difference of the initial impactor velocity between FV and FM cases under the same impact energy becomes apparent. Since less compression is needed for a larger initial impactor velocity under the same impact energy, the maximum displacement of the impactor for FV case is always larger than that for FM case and the difference increases with the growth of the impact energy from $E_{0}$ to $6 E_{0}$. Then with the continued increment of the impact energy this difference narrows because the buckypaper in FV case reaches its deformation limit whereas the buckypaper in FM cases still has deformation capability left.

The difference between the initial kinetic energy of the impactor $\left(E_{\text {initial }}\right)$ and the final kinetic energy after detachment $\left(E_{\text {final }}\right)$ is defined as energy dissipation of the impactor. Based on the principle of energy conservation, the energy dissipation of the impactor equals to the energy absorption by the buckypaper. That is, the dissipated kinetic energy of the impactor is transformed into the strain energy induced by the deformation and thermal energy induced by the frictional sliding between CNTs and plastic deformation. A larger impact velocity is favorable to introduce more sliding between CNTs thus creating more thermal energy, which explains the phenomenon that less compression distance is needed for the FM cases under the same impact energy from the energetic standpoint. According to Figure 8, the increase of the linear center-ofmass kinetic energy of the buckypaper due to the impact is very tiny in both FM and FV cases so that the increased kinetic energy of the buckypaper can be regarded as almost completely transformed into thermal energy. In the FM cases the energy dissipation keeps increasing nearly linearly as the impact energy rises from $E_{0}$ to $10 E_{0}$. Whereas in the FV cases this linear trend remains only from $E_{0}$ to $6 E_{0}$ because the buckypaper deformation becomes saturated at the 
impact energy $6 E_{0}$. Slight squeezing deformation and sliding between CNTs still exist in a saturated buckypaper subjected to impact, therefore for impact energy larger than $6 E_{0}$ the energy absorption still increases with a larger impact energy, but the rate of increase lessens at a higher impact energy since the stiffening buckypaper at the final stage of impact attenuates energy absorption capability. It is worthwhile to mention that the slope of the linear increment stage in the FM curve is higher than that in the FV curve. That means, more impact energy is absorbed by the buckypaper in the FM case than in the FV case even under the same impact energy, owing to the less recoverable deformation of the buckypaper in the FM cases. Based on the ratio of the energy dissipation to the initial impact energy, the energy dissipation efficiency as interpreted in Figure 8 can be acquired by the formula $\left(E_{\text {initial }}-E_{\text {final }}\right) / E_{\text {initial }}$. It is found that the buckypaper shows remarkable energy dissipation efficiency for a specific range of impact energy. For the FV cases, with the growth of the impact energy the energy dissipation efficiency is always up to about $96.5 \%$ before the deformation of the buckypaper is oversaturated, followed by a fast decline of the efficiency corresponding to the nonlinear increase of the energy absorption. For the FM cases, the energy dissipation efficiency at the impact energy $E_{0}$ is similar to that of the FV case because of the similar impact velocities. As the impact energy increases to $3 E_{0}$, the efficiency improves to $99.52 \%$ and then with successive growth of the impact energy the efficiency remains rising slowly to $100 \%$. This finding indicates that the increase of the energy absorption for FM cases is not strictly linear. In fact, the increased rate of energy absorption is improved slightly with the increment of the impact energy. The reason is that a larger impact velocity causes more sliding between CNTs and thus generates a higher ratio of thermal energy to the total dissipation energy, leading to a relatively lower ratio of recoverable impact energy to the initial impact energy. 


\subsection{Effects of density of buckypaper}

In terms of the effects of the buckypaper density on the dynamic behavior and energy dissipation capability of the buckypaper, computational specimens consisting of 512 SWCNTs with the same length of $100 \mathrm{~nm}$ occupy different volumes of simulation boxes. Impact energy is changed by FM method. According to Goldsmith [75], the mechanism involving permanent deformation can no longer be described by the equations of the elastic domain, but must be interpreted by relations capable of including the large strains and permanent deformations encountered in the impact process. The theory of plastic wave propagation or plastic flow always postulates incompressibility of the material in the plastic domain which requires invariance of the bulk modulus, while hydrodynamic theory can be used to describe this impact problem involving compressible medium. The hydrodynamic theory of wave propagation treats the medium buckypaper as a compressible fluid without shear resistance and, to a first approximation, without viscosity. By neglecting the shear modulus of the material, all permanent deformations are considered to occur as the result of changes in the bulk modulus or, equivalently, in the density of the body. The hydrodynamic theory consider that the motion of the particles behind the shock front to be steady, however, in our research the impact behavior between the impactor and the buckypaper keeps for an extended period. That is, behind the shock front the continuous compression keeps going until the impactor departs from the buckypaper, which causes the impact problem to be more complicated. And neglecting the effects of the porosity of the buckypaper, here we just focus on the initial shock stage to calculate the velocity of the shock front for a rough estimation. Once the impactor strikes the upper surface of the buckypaper, the constituent particles of the upper surface instantaneously have the same velocity as the impactor does, creating a violent shock wave - the Hugoniot shock - that travels along the thickness of 
buckypaper and brings the adjacent particles to have the same velocity. The shocked region behind the wave is subject to a transient Hugoniot pressure of high magnitude, which explains the rapid increase of the contact force at the initial stage in Figure 4. Based on the laws of conservation of mass, momentum, and energy, the governing equation for this shock process can be expresses as: $\rho_{1}\left(U-v_{1}\right)=\rho_{0}\left(U-v_{0}\right), \quad p_{1}-p_{0}=\rho_{0}\left(U-v_{0}\right)\left(v_{1}-v_{0}\right)$, and $p_{1} v_{1}-$ $p_{0} v_{0}=\rho_{0}\left(U-v_{0}\right)\left[I_{1}-I_{0}+\frac{1}{2}\left(v_{1}^{2}-v_{0}^{2}\right)\right]$, where subscripts 0 and 1 denote the undisturbed and compressed state of the buckypaper, $\rho$ is the density of the buckypaper, $p$ is the shock pressure and gives the maximum possible value for the impact at its very beginning, $v$ is the particle velocity, $U$ is the shock wave velocity and $I$ represents the internal energy per unit mass. Because the buckypaper is initially at rest and free, $v_{0}=0$ and $p_{0}=0$. Thus the shock wave velocity can be expressed as $U=\frac{p_{1}}{\rho_{0} v_{1}}$, where $v_{1}$ and $p_{1}$ can be derived from the impactor velocity and the impactor-buckypaper interaction respectively. The shock wave velocities in buckypapers with different buckypapers derived from hydrodynamic theory and tracked from the simulations are presented in Figure S2. Since the sound speed in the uncompressed state is identical to the velocity of the propagation of elastic compressional waves in the medium, the elastic compressional wave velocity can be derived based on the Newton-Laplace equation $c=\sqrt{\left(K+\frac{4}{3} G\right) / \rho}$, where $K$ and $G$ denoting the bulk modulus and shear modulus of the buckypaper respectively are obtained from MD simulations of compression and shear tests (shown in Figure S5). The speed sound is also shown in Figure S2. It is found that the real compressional wave velocity is much higher than the elastic one because of the compressibility of the buckypaper, which agrees well with the results in Reference [75]. Since the receiver is 
fixed and rigid, enforcing zero-displacement normal to the boundary, the wave is reflected completely without energy loss.

Figure 9 presents the energy dissipation efficiency of the buckypaper with different densities versus impact energy. For low impact energy, energy dissipation efficiencies for all cases are extremely high, about $95 \%$ to $98 \%$, due to its sufficient deformation capability. On this occasion the buckypaper with a lower density seems to have slightly higher energy dissipation efficiency owing to the lower stiffness of the buckypaper. As the impact energy rises, the efficiency declines first for the lowest-density buckypaper, followed by higher-density buckypapers in turn. That is, buckypapers with a higher density are capable of bearing higher impact energies within the deformation limit. Thus in the case that the buckypaper has oversaturated deformation, a higher-density buckypaper has a higher energy dissipation efficiency under the same impact energy. For densities $40.67 \mathrm{~kg} / \mathrm{m}^{3}, 44.38 \mathrm{~kg} / \mathrm{m}^{3}$ and $52.81 \mathrm{~kg} / \mathrm{m}^{3}$, it can be inferred that the decline of energy dissipation efficiency begins under impact energies between $4 E_{0}$ to $5 E_{0}$ in order and the efficiencies under the same impact energy are close to each other due to their similar densities. The buckypaper with the largest density can sustain the impact energy up to $8 E_{0}$ within the deformation limit. It is because that a larger density means more sliding occurred and thus more thermal energy dissipated.

Figure 10 traces the upper contact force versus the compression distance at the impact energy $4 E_{0}$ and $7 E_{0}$ respectively. It can be found that the maximum compression distance declines with the growth of the density. Because at the same compression distance a larger density means more CNTs are deformed and more sliding occurs between CNTs to produce more strain energy and more thermal energy. Snapshots (a) and (b) in Figure 10 which show the morphology of buckypapers with density $78.97 \mathrm{~kg} / \mathrm{m}^{3}$ and $40.67 \mathrm{~kg} / \mathrm{m}^{3}$ respectively at the maximum 
compression distance under impact energy $4 E_{0}$ show that at the current impact energy the buckypapers with four different densities possess sufficient deformation capability at the largest compression. At the initial stage the peak force for the higher-density buckypaper is larger due to the increased stiffness of the deformed region. However, the second peaks nearby the maximum compression distance for the above four cases are approximately the same due to the similar stiffness resulting from the combination of compression distance and density. Snapshots (c), (d) and (e) show the morphologies of the buckypaper with densities of $78.97 \mathrm{~kg} / \mathrm{m}^{3}, 62.05 \mathrm{~kg} / \mathrm{m}^{3}$ and $40.67 \mathrm{~kg} / \mathrm{m}^{3}$ respectively at the maximum compression distance under impact energy $7 E_{0}$. They indicate that impact energy $7 E_{0}$ enables the buckypaper with density $78.97 \mathrm{~kg} / \mathrm{m}^{3}$ to just achieve the deformation limit but the buckypaper with a lower density experiences oversaturated deformation in accordance with the results in Figure 9. Therefore, the force increases suddenly when the impactor approaches the maximum compression and the peak ascends sharply with the decline of the density. Figure 11 presents the maximum upper contact force versus the density under different impact energies. For the impact energy varying from $E_{0}$ to $4 E_{0}$, all the buckypapers with different densities possess adequate energy deformation capability. As a result, as the density grows the maximum contact forces have no obvious difference and as the impact energy grows the force shows a slight increment. As the impact energy increases from $5 E_{0}$ to $8 E_{0}$, oversaturated deformation first appears in a lower-density buckypaper resulting in the abrupt increase of the force, whereas for the higher-density buckypaper the force remains steady. For the impact energy over a critical value, oversaturated deformation appears in all the buckypapers and thus the force declines with the growth of the density.

As discussed earlier, the compression distance of the buckypaper is influenced by the contact force and the energy dissipation. Since a larger density typically corresponds to a smaller 
specimen size of the buckypaper, the compression ratio is defined as the percentage of the maximum compression distance to the height of the buckypaper. The relationship among the compression ratio, density and impact energy is given in Figure 12. For a certain density, as the impact energy grows the compression ratio increases with a declining rate and eventually tends to be constant due to the oversaturated deformation. For certain impact energy, with the increment of the density both the compression ratio and the maximum compression distance decline. It means that under the same compression ratio more energy is absorbed by the buckypaper with a larger density mainly due to more sliding between CNTs dissipating more kinetic energy into thermal energy. At the lowest impact energy, the decline of the compression ratio versus the density is slight. As impact energy raises, the decline rate increases first and then decreases with the appearance of the oversaturated deformation of the buckypaper. In addition, since the buckypaper with a low density reaches the deformation limit at the low impact energy, the compression ratio also reaches stable. It can be found that at the largest impact energy only the buckypaper with the largest density $107.6 \mathrm{~kg} / \mathrm{m}^{3}$ still has slight room for compression and the compression ratios for different densities are very close.

Figure 13 depicts the energy absorption per unit volume (EAUV) of the buckypaper. With the increment of the density, the EAUV increases linearly with the impact energy until oversaturated deformation, such as $E_{0}$ and $3 E_{0}$. This can be probably explained as the number of beads per unit volume is proportional to the density of the buckypaper. The slope can be calculated by $\left(\Delta E_{h}-\Delta E_{l}\right) /\left(d_{h}-d_{l}\right)$ where $d_{h}$ and $d_{l}$ are the higher and lower density respectively and $\Delta E_{h}$ and $\Delta E_{l}$ are the energy dissipation corresponding to $d_{h}$ and $d_{l}$ respectively. As is known from Figure 8 , for the impact energy that does not cause the oversaturated deformation of the buckypaper, the energy absorption as well as EAUM is proportional to the impact energy at a 
certain density. As a result, the slope is also proportional to the impact energy approximately. As the impact energy rises, the lower-density buckypaper reaches its deformation limit more quickly and thus the increment of EAUV versus impact energy becomes slower, resulting in nonlinear increment of the EAUV versus density.

\subsection{Effects of length of SWCNT}

Since individual length distribution of SWCNT can vary from several nanometers to micrometers and even meters [69], it is noteworthy to investigate the effect of the individual CNTs' length on the energy dissipation capability of the buckypaper. Due to the limit of the computational resource, the lengths of individual SWCNTs used here are in the range of 100nm$800 \mathrm{~nm}$. Figure 14 presents the energy dissipation efficiency of the buckypaper with different lengths of individual SWCNTs from $100 \mathrm{~nm}, 149 \mathrm{~nm}, 237 \mathrm{~nm}, 410 \mathrm{~nm}$ to $800 \mathrm{~nm}$ at different impact energies. Densities of all the specimens in this section are kept at $78.97 \mathrm{~kg} / \mathrm{m}^{3}$, the same as that in Section 3.1. It can be found that the energy dissipation efficiency varies slightly as the length of individual SWCNTs changes. For impact energies $E_{0}$ and $4 E_{0}$, the efficiency always remains about 96\%. As is discussed in Section 3.1, the buckypaper with SWCNTs of length $100 \mathrm{~nm}$ does not reach its deformation limit under both impact energies. It can be inferred that buckypapers studies in this section are all below their deformation limits. For the impact energy $7 E_{0}$, the efficiency is slightly lower than that for $E_{0}$ and $4 E_{0}$, which indicates that the buckypaper has slightly saturated deformation under impact energy $7 E_{0}$. For impact energy $10 E_{0}$, the efficiency falls greatly to around $86 \%$ due to the limited energy absorption capability of buckypaper after reaching its deformation limit. These findings imply that the energy dissipation efficiency is independent of the length of individual SWCNTs. Likewise, Figure 15 shows that the maximum compression distance of the buckypaper remains almost unchanged 
with different lengths of individual SWCNTs under the four impact energies. The difference is that the maximum compression distance increases greatly from the impact energy $E_{0}$ to $4 E_{0}$ while it increases gently from impact energy $4 E_{0}$ to $7 E_{0}$. The reason is that as the compression distance grows the density of the buckypaper increases, and thus further compression with the same distance is able to absorb more impact energy. The maximum compression distance increases very slightly for impact energy from $7 E_{0}$ to $10 E_{0}$ since the buckypaper reaches its deformation limit. Figure 16 illustrates that the maximum upper contact force almost remains constant when the length of individual SWCNTs varies. The force increases slightly for impact energies from $E_{0}$ to $4 E_{0}$ due to the maximum compression distance being far away from the limit. Then an obvious increment of the force happens for impact energies from $4 E_{0}$ to $7 E_{0}$ due to the slightly saturated deformation corresponding to impact energy $7 E_{0}$. Then the force increases tremendously for impact energy from $7 E_{0}$ to $10 E_{0}$ as the buckypaper reaches the deformation limit. Hence, it can be inferred that at the same impact energy the evolution of the contact force follows the compression distance irrespective of the variation of the length of individual SWCNTs, since the energy dissipation efficiency, the maximum compression distance, and the maximum contact force are all independent of the length of individual SWCNTs. The reason is that the effects of the length on pore size can be negligible [56] and the mechanical properties of such a buckypaper mainly depend on the mass density of the buckypaper, which has been reported in both experiment and simulation [58]. By tracking energy variation during the impact process (shown in Table S1), it is found that in the initial state the angle energy increases with the increment of the length of individual SWCNTs, since longer SWCNTs are more prevalent to buckling. However, the variations of angle energy corresponding to different lengths due to the impact are almost the same. It also can be found that the thermal 
energy takes major part of the absorbed energy, that is, the dominant mechanism is internal sliding.

\section{Conclusions}

In this paper, the dynamic behavior and energy dissipation capability of a buckypaper have been extensively investigated by performing a series of molecular dynamics simulations with a variety of design parameters such as impact energy, the density of buckypaper, and the length of individual SWCNTs. Effects of these parameters on energy dissipation capability of the buckypaper have been analyzed. Simulation results indicate that the buckypaper possesses extremely high energy dissipation efficiency, always more than $95 \%$, before reaching its deformation limit. Under the same impact energy, a larger impact velocity yields slightly higher energy dissipation efficiency, even close to $100 \%$, owing to more sliding between SWCNTs and thus more energy dissipated into thermal energy. When the buckypaper exceeds its deformation limit, the energy dissipation efficiency declines, and both the upper and lower contact forces increase abruptly. Within the deformation limit, the buckypaper with higher density is able to sustain larger impact energies due to the increment of more strain energy and thermal energy under the same compression but has slightly lower energy dissipation efficiency due to the increased compression stiffness. Therefore, for specific impact energy it is feasible to adjust the volume of the buckypaper by tuning the density within certain realms to fulfill the desired requirements. It is also found that the energy dissipation capability of a buckypaper is independent on the length of individual SWCNTs since the contact forces follows the evolution of the compression distance while changing the length of individual SWCNTs. It has to be noted that the lengths used in this paper are far below the persistence length, large systems with extremely long CNTs is likely to behave much differently. Besides the remarkable energy 
dissipation efficiency, the buckypaper also reveals remarkable mass density and volume density of energy dissipation up to $90 \mathrm{~J} / \mathrm{g}$ and $8.6 \mathrm{~J} / \mathrm{cm}^{3}$ respectively which may be further improved by enlarging the impact energy and/or changing its density. Thus the buckypaper is applicable for volume-controlled and mass-controlled products essential to be protected from impact. Overall, in spite that all these findings are obtained in silico, this study can shed lights on the dynamic behavior of the buckypaper, offering a promising candidate for energy dissipation devices and stimulating more relevant work.

No research, however, provides a perfect study, with this work being no exception to the rule. More endeavors should be devoted to expand the realm of the length of individual CNTs, the impact energy, the impact velocity under the same impact energy and the density of buckypaper. Particularly, the buckypaper density can be obtained as large as $1.39 \mathrm{~g} / \mathrm{cm}^{3}$, thus yielding greatly improved transport and mechanical properties such as Young's modulus over $2 \mathrm{GPa}$ [54]. Fluid component can be introduced within the pores which would provide a hydraulic action and viscoelastic drag[77]. Besides, previous research has proved that mixture of SWCNTs with double-walled carbon nanotubes (DWCNTs) in a buckypaper influences its ultimate mechanical property due to the strong adhesion energy and large bending rigidity [56]. Especially, the Poisson's ratio of the mixed buckypaper can be tuned from a positive to a negative value by increasing the DWCNT composition, which is beneficial for the energy dissipation. Therefore, mixed buckypaper should be explored to achieve better energy dissipation capability. In addition, other forms of buckypaper could be set up, such as that made of aligned CNTs which has better performance on thermal and electrical conductance [78], and cross-links between CNTs which enhances thermal stability and load transfer of buckypaper [79]. Besides the uniaxial compressive loading, there are many other forms loading and deformation mechanisms pertinent 
to collisions, impact and penetration, such as membrane response of buckypaper at high frequency vibration [80], the tensile and fracture response in the case of spall [81] and the bending response of buckypaper [82]. Since the reflected wave is determined by the nature of the boundary condition [83], other types of reflecting boundary are worthwhile to be invested in the future, such as free surface boundary which has zero-stress conditions and allows for spall in the case of tension at the away-boundary as well as compression near the impact boundary. Other types, not limited, include elastic boundary and attached dashpot, corresponding to more complicated wave reflection. The energy dissipation and dynamic response of the buckypaper with different boundaries would likely be much different from each other. 


\section{Acknowledgements}

HC, JC, and HN appreciate the support from the Scholarship of Nanjing University of Aeronautics and Astronautics (China). LZ, MB, and XW would like to acknowledge support from the University of Georgia (UGA) Research Foundation. Calculations are performed at the UGA Advanced Computing Resource Centre.

\section{Author information}

Corresponding author: Xianqiao Wang

Email: xqwang@uga.edu.

Competing financial interests: The authors declare no competing financial interests. 


\section{References}

[1] Lu G, Yu TX. Energy Absorption of Structures and Materials: Elsevier; 2003.

[2] Ramakrishna S. Microstructural design of composite materials for crashworthy structural applications. Materials \& Design. 1997;18(3):167-73.

[3] Verlinden B. Severe plastic deformation of metals. MJoM. 2005;11:165-82.

[4] Banhart J. Manufacture, characterisation and application of cellular metals and metal foams. Progress in materials science. 2001;46(6):559-632.

[5] Ashby MF, Evans T, Fleck NA, Hutchinson J, Wadley H, Gibson L. Metal Foams: A Design Guide: A Design Guide: Elsevier; 2000.

[6] Zhao H, Gary G. CRUSHING BEHAVIOUR OF ALUMINIUM HONEYCOMBS

UNDER IMPACT LOADING. International Journal of Impact Engineering. 1998;21(10):82736.

[7] Ruan D, Lu G, Wang B, Yu T. In-plane dynamic crushing of honeycombs - a finite element study. International Journal of Impact Engineering. 2003;28(2):161-82.

[8] CHEN J-b, NIE H, ZHAO J-c, BAI H-m, BO W. Research of the Factors of Buffering Performance in Lunar Lander [J]. Journal of Astronautics. 2008;6:010.

[9] Nemat-Nasser S, Kang W, McGee J, Guo W-G, Isaacs J. Experimental investigation of energy-absorption characteristics of components of sandwich structures. International journal of impact engineering. 2007;34(6):1119-46.

[10] Hazizan MA, Cantwell WJ. The low velocity impact response of an aluminium honeycomb sandwich structure. Composites Part B: Engineering. 2003;34(8):679-87.

[11] Zhang L, Becton M, Wang X. Mechanical analysis of graphene-based woven nanofabric. Materials Science and Engineering: A. 2015;620:367-74.

[12] Chandra R, Singh SP, Gupta K. Damping studies in fiber-reinforced composites - a review. Composite Structures. 1999;46(1):41-51.

[13] Sun CT, Chaturvedi SK, Gibson RF. Special Issue: Advances and Trends in Structures and Dynamics Internal damping of short-fiber reinforced polymer matrix composites. Computers \& Structures. 1985;20(1):391-400.

[14] Zhang J, Wang X, Lu L, Li D, Yang X. Preparation and performance of high-impact polystyrene (HIPS)/nano-TiO2 nanocomposites. Journal of applied polymer science. 2003;87(3):381-5.

[15] Sun L, Gibson RF, Gordaninejad F, Suhr J. Energy absorption capability of nanocomposites: A review. Composites Science and Technology. 2009;69(14):2392-409.

[16] Viana JC. Polymeric materials for impact and energy dissipation. Plastics, rubber and composites. 2006;35(6-7):260.

[17] Surani FB, Kong X, Panchal DB, Qiao Y. Energy absorption of a nanoporous system subjected to dynamic loadings. Applied Physics Letters. 2005;87(16):163111. 
[18] Xu B, Liu L, Zhou Q, Qiao Y, Xu J, Li Y, et al. Energy Dissipation of Nanoporous MFI Zeolite Under Dynamic Crushing. Journal of Computational and Theoretical Nanoscience. 2011;8(5):881-6.

[19] Winter N, Becton M, Zhang L, Wang X. Failure Mechanisms and Scaling Laws of Nanoporous Aluminum: A Computational Study Advanced Engineering Materials. 2015:n/an/a.

[20] Han A, Punyamurthula VK, Lu WY, Qiao Y. Deformation of a nanoporous silica under compressive loading. J Appl Phys. 2008;103(8):5.

[21] Cao G. Working mechanism of nanoporous energy absorption system under high speed loading. The Journal of Physical Chemistry C. 2012;116(14):8278-86.

[22] Chen X, Surani FB, Kong X, Punyamurtula VK, Qiao Y. Energy absorption performance of steel tubes enhanced by a nanoporous material functionalized liquid. Applied physics letters. 2006;89(24):241918.

[23] Liu H, Cao G. Interaction between mechanical wave and nanoporous energy absorption system. The Journal of Physical Chemistry C. 2013;117(8):4245-52.

[24] Punyamurtula VK, Han A, Qiao Y. An experimental investigation on a nanoporous carbon functionalized liquid damper. Philos Mag Lett. 2006;86(12):829-35.

[25] Qiao Y, Kong X, Surani FB. Nanoporous Energy Absorption Systems. ASME 4th Integrated Nanosystems Conference: American Society of Mechanical Engineers; p. 3-4.

[26] Chen X, Huang Y. Nanomechanics modeling and simulation of carbon nanotubes. Journal of Engineering Mechanics. 2008;134(3):211-6.

[27] Delfani MR, Shodja HM, Ojaghnezhad F. Mechanics and morphology of single-walled carbon nanotubes: from graphene to the elastica. Philosophical Magazine. 2013;93(17):2057-88.

[28] Salvetat JP, Bonard JM, Thomson NH, Kulik AJ, Forró L, Benoit W, et al. Mechanical properties of carbon nanotubes. Appl Phys A. 1999;69(3):255-60.

[29] Wang CM, Zhang YY, Xiang Y, Reddy JN. Recent Studies on Buckling of Carbon Nanotubes. Applied Mechanics Reviews. 2010;63(3).

[30] Becton M, Zhang L, Wang X. Molecular Dynamics Study of Programmable Nanoporous Graphene. Journal of Nanomechanics and Micromechanics. 2014;4(3).

[31] Zhang Z, Wang X, Li J. Simulation of collisions between buckyballs and graphene sheets. International Journal of Smart and Nano Materials. 2012;3(1):14-22.

[32] Zhang L, Wang X. Atomistic insights into the nanohelix of hydrogenated graphene: formation, characterization and application. Phys Chem Chem Phys. 2014;16(7):2981-8.

[33] Zhang L, Wang X. Tailoring Pull-out Properties of Single-Walled Carbon Nanotube Bundles by Varying Binding Structures through Molecular Dynamics Simulation. J Chem Theory Comput. 2014;10(8):3200-6.

[34] Treacy MMJ, Ebbesen TW, Gibson JM. Exceptionally high Young's modulus observed for individual carbon nanotubes. Nature. 1996;381(6584):678-80. 
[35] Peng B, Locascio M, Zapol P, Li SY, Mielke SL, Schatz GC, et al. Measurements of near-ultimate strength for multiwalled carbon nanotubes and irradiation-induced crosslinking improvements. Nat Nanotechnol. 2008;3(10):626-31.

[36] Xu J, Li Y, Xiang Y, Chen X. Energy absorption ability of buckyball C-720 at low impact speed: a numerical study based on molecular dynamics. Nanoscale Research Letters. 2013;8:1-10.

[37] Xu J, Li Y, Xiang Y, Chen X. A Super Energy Mitigation Nanostructure at High Impact Speed Based on Buckyball System. Plos One. 2013;8(5).

[38] Xu J, Sun Y, Wang B, Li Y, Xiang Y, Chen X. Molecular dynamics simulation of impact response of buckyballs. Mechanics Research Communications. 2013;49:8-12.

[39] Chen H, Zhang L, Becton MD, Nie H, Chen J, Wang X. Molecular Dynamic Study of a CNT/Buckyballs-enabled Energy Absorption System. Physical Chemistry Chemical Physics. 2015;17:17311-21.

[40] Weidt D, Buggy M. Prediction of energy absorption characteristics of aligned carbon nanotube/epoxy nanocomposites. IOP Conference Series: Materials Science and Engineering: IOP Publishing; p. 012028.

[41] Thostenson ET, Ren ZF, Chou TW. Advances in the science and technology of carbon nanotubes and their composites: a review. Composites Science and Technology. 2001;61(13):1899-912.

[42] Wang X, Wang X, Hu L. Synthesis of CNT/In2O3 Nanocomposite by Sol-Gel Method and Its Photocatalytic Property. Asian Journal of Chemistry. 2013;25(10):5798-800.

[43] Zhou X, Shen L, Li L, Zhou S, Huang T, Hu C, et al. Microwave sintering carbon nanotube/Ni0.5Zn0.5Fe2O4 composites and their electromagnetic performance. Journal of the European Ceramic Society. 2013;33(11):2119-26.

[44] Gui X, Zeng Z, Zhu Y, Li H, Lin Z, Gan Q, et al. Three-Dimensional Carbon Nanotube Sponge-Array Architectures with High Energy Dissipation. Advanced Materials. 2014;26(8):1248-53.

[45] Lattanzi L, De Nardo L, Raney JR, Daraio C. Geometry-Induced Mechanical Properties of Carbon Nanotube Foams. Advanced Engineering Materials. 2014;16(8):1026-31.

[46] Liu LQ, Ma WJ, Zhang Z. Macroscopic Carbon Nanotube Assemblies: Preparation, Properties, and Potential Applications. Small. 2011;7(11):1504-20.

[47] Rinzler AG, Liu J, Dai H, Nikolaev P, Huffman CB, Rodriguez-Macias FJ, et al. Largescale purification of single-wall carbon nanotubes: process, product, and characterization. Appl Phys A-Mater Sci Process. 1998;67(1):29-37.

[48] Thess A, Lee R, Nikolaev P, Dai H, Petit P, Robert J, et al. Crystalline ropes of metallic carbon nanotubes. Science-AAAS-Weekly Paper Edition. 1996;273(5274):483-7.

[49] Hall LJ, Coluci VR, Galvão DS, Kozlov ME, Zhang M, Dantas SO, et al. Sign change of Poisson's ratio for carbon nanotube sheets. Science. 2008;320(5875):504-7. 
[50] Romo-Herrera JM, Terrones M, Terrones H, Meunier V. Guiding electrical current in nanotube circuits using structural defects: a step forward in nanoelectronics. ACS nano. 2008;2(12):2585-91.

[51] Li Y, Qiu X, Yang F, Yin Y, Fan Q. Stretching-dominated deformation mechanism in a super square carbon nanotube network. Carbon. 2009;47(3):812-9.

[52] Li Y, Qiu X, Yang F, Wang X-S, Yin Y. The effective modulus of super carbon nanotubes predicted by molecular structure mechanics. Nanotechnology. 2008;19(22):225701.

[53] Zsoldos I, Laszlo I. Computation of the loading diagram and the tensile strength of carbon nanotube networks. Carbon. 2009;47(5):1327-34.

[54] Zhang L, Zhang G, Liu C, Fan S. High-Density Carbon Nanotube Buckypapers with Superior Transport and Mechanical Properties. Nano Letters. 2012;12(9):4848-52.

[55] Cranford SW. In silico assembly and nanomechanical characterization of carbon nanotube buckypaper. Nanotechnology. 2010;21(26):265706.

[56] Li Y, Kroeger M. A theoretical evaluation of the effects of carbon nanotube entanglement and bundling on the structural and mechanical properties of buckypaper. Carbon.

2012;50(5):1793-806.

[57] Xie B, Liu Y, Ding Y, Zheng Q, Xu Z. Mechanics of carbon nanotube networks: microstructural evolution and optimal design. Soft Matter. 2011;7(21):10039-47.

[58] Yang X, He P, Gao H. Modeling frequency-and temperature-invariant dissipative behaviors of randomly entangled carbon nanotube networks under cyclic loading. Nano Research. 2011;4(12):1191-8.

[59] Xu M, Futaba DN, Yamada T, Yumura M, Hata K. Carbon nanotubes with temperatureinvariant viscoelasticity from-196 to 1000 C. Science. 2010;330(6009):1364-8.

[60] Li Y, Kroeger M. Viscoelasticity of carbon nanotube buckypaper: zipping-unzipping mechanism and entanglement effects. Soft Matter. 2012;8(30):7822-30.

[61] Whitby RLD, Fukuda T, Maekawa T, James SL, Mikhalovsky SV. Geometric control and tuneable pore size distribution of buckypaper and buckydiscs. Carbon. 2008;46(6):949-56.

[62] Wu Q, Zhu W, Zhang C, Liang Z, Wang B. Study of fire retardant behavior of carbon nanotube membranes and carbon nanofiber paper in carbon fiber reinforced epoxy composites. Carbon. 2010;48(6):1799-806.

[63] Zhou W, Huang Y, Liu B, Hwang KC, Zuo JM, Buehler MJ, et al. Self-folding of singleand multiwall carbon nanotubes. Applied Physics Letters. 2007;90(7):073107.

[64] Plimpton S. Fast Parallel Algorithms for Short-Range Molecular Dynamics. Journal of Computational Physics. 1995;117(1):1-19.

[65] Wang C, Wang L, Xu Z. Enhanced mechanical properties of carbon nanotube networks by mobile and discrete binders. Carbon. 2013;64:237-44.

[66] Li Y, Kroger M. Computational study on entanglement length and pore size of carbon nanotube buckypaper. Applied Physics Letters. 2012;100(2). 
[67] Chenoweth K, van Duin ACT, Goddard WA. ReaxFF Reactive Force Field for Molecular Dynamics Simulations of Hydrocarbon Oxidation. The Journal of Physical Chemistry A. 2008;112(5):1040-53.

[68] van Duin ACT, Dasgupta S, Lorant F, Goddard WA. ReaxFF: A Reactive Force Field for Hydrocarbons. The Journal of Physical Chemistry A. 2001;105(41):9396-409.

[69] Buehler MJ. Mesoscale modeling of mechanics of carbon nanotubes: Self-assembly, selffolding, and fracture. Journal of Materials Research. 2006;21(11):2855-69.

[70] Cranford S, Yao H, Ortiz C, Buehler MJ. A single degree of freedom 'lollipop' model for carbon nanotube bundle formation. Journal of the Mechanics and Physics of Solids. 2010;58(3):409-27.

[71] Liu Q, Ren W, Wang D-W, Chen Z-G, Pei S, Liu B, et al. In Situ Assembly of MultiSheeted Buckybooks from Single-Walled Carbon Nanotubes. Acs Nano. 2009;3(3):707-13.

[72] Xu G, Zhang Q, Zhou W, Huang J, Wei F. The feasibility of producing MWCNT paper and strong MWCNT film from VACNT array. Appl Phys A. 2008;92(3):531-9.

[73] Berhan L, Yi Y, Sastry A, Munoz E, Selvidge M, Baughman R. Mechanical properties of nanotube sheets: Alterations in joint morphology and achievable moduli in manufacturable materials. J Appl Phys. 2004;95(8):4335-45.

[74] Stukowski A. Visualization and analysis of atomistic simulation data with OVITO-the Open Visualization Tool. Modelling and Simulation in Materials Science and Engineering. 2010;18(1):015012.

[75] Goldsmith W. Impact: Courier Corporation; 2001.

[76] Krehl PO. History of shock waves, explosions and impact: a chronological and biographical reference: Springer Science \& Business Media; 2008.

[77] Saada MA, Rigolet S, Paillaud J-L, Bats N, Soulard M, Patarin J. Investigation of the energetic performance of pure silica ITQ-4 (IFR) zeolite under high pressure water intrusion. The Journal of Physical Chemistry C. 2010;114(26):11650-8.

[78] Wang D, Song P, Liu C, Wu W, Fan S. Highly oriented carbon nanotube papers made of aligned carbon nanotubes. Nanotechnology. 2008;19(7):075609.

[79] Chen I-WP, Liang R, Zhao H, Wang B, Zhang C. Highly conductive carbon nanotube buckypapers with improved doping stability via conjugational cross-linking. Nanotechnology. 2011;22(48):485708.

[80] Bouthier OM, Bernhard RJ. Simple models of energy flow in vibrating membranes. Journal of Sound and Vibration. 1995;182(1):129-47.

[81] Schuler H, Mayrhofer C, Thoma K. Spall experiments for the measurement of the tensile strength and fracture energy of concrete at high strain rates. International Journal of Impact Engineering. 2006;32(10):1635-50.

[82] Volkov AN, Zhigilei LV. Structural Stability of Carbon Nanotube Films: The Role of Bending Buckling. Acs Nano. 2010;4(10):6187-95. 
[83] Ozden S, Narayanan TN, Tiwary CS, Dong P, Hart AHC, Vajtai R, et al. 3D Macroporous Solids from Chemically Cross-linked Carbon Nanotubes. Small. 2015;11(6):68893. 
Table 1 Potential parameters for the coarse-grained model of $(5,5) \mathrm{SWCNT}$

\begin{tabular}{lc}
\hline Parameters & $(5,5) \mathrm{SWCNT}$ \\
\hline Equilibrium bead distance, $\boldsymbol{r}_{\mathbf{0}}(\AA)$ & 10 \\
Stretching constant, $\boldsymbol{k}_{\boldsymbol{b}}\left(\mathbf{k c a l} /\left(\mathbf{m o l} \cdot \AA^{\mathbf{2}}\right)\right)$ & 1,000 \\
Equilibrium angle, $\boldsymbol{\theta}_{\mathbf{0}}\left(^{\circ}\right)$ & 180 \\
Bending constant, $\boldsymbol{k}_{\boldsymbol{a}}\left(\mathbf{k c a l} /\left(\mathbf{m o l} \cdot \mathbf{r a d}^{2}\right)\right)$ & 14,300 \\
vdW distance, $\boldsymbol{\sigma}(\AA)$ & 9.35 \\
vdW energy, $\boldsymbol{\varepsilon}(\mathbf{k c a l} / \mathbf{m o l})$ & 15.10 \\
\hline
\end{tabular}




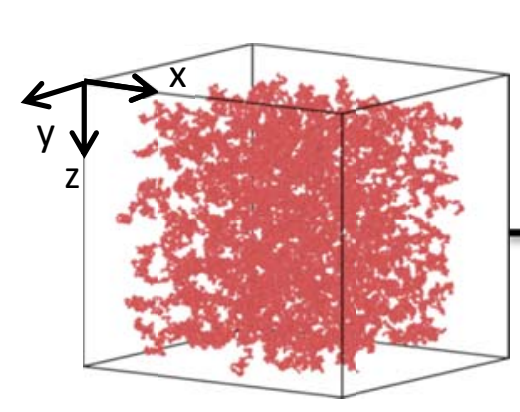

(a) Random walk

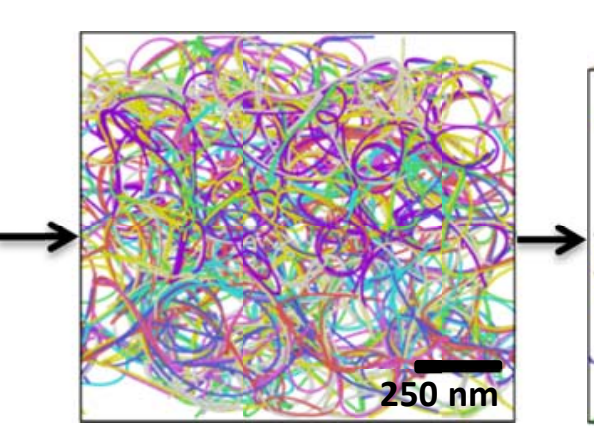

(b) Buckypaper

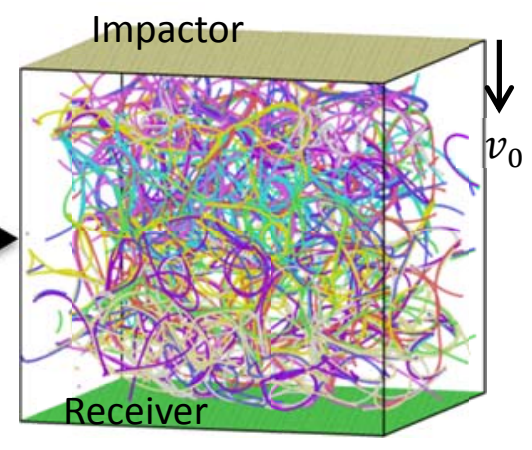

(c) Computational model

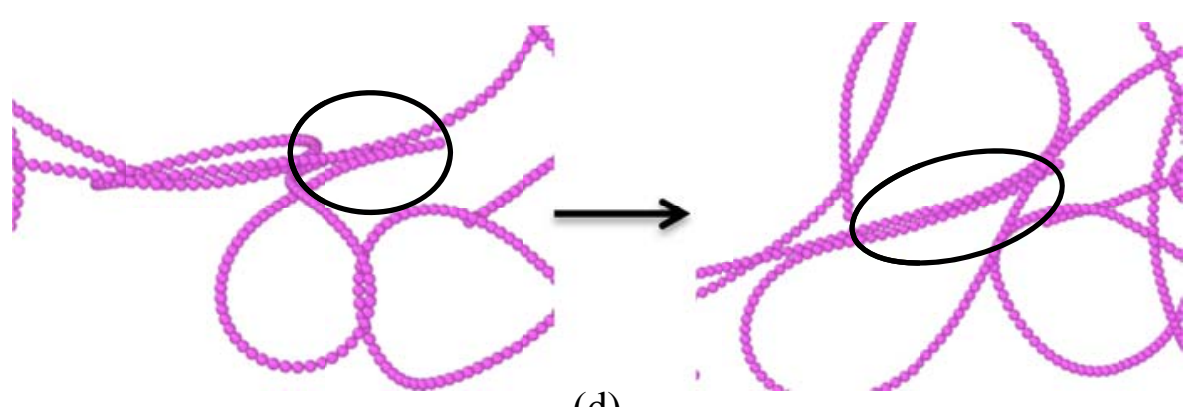

(d)

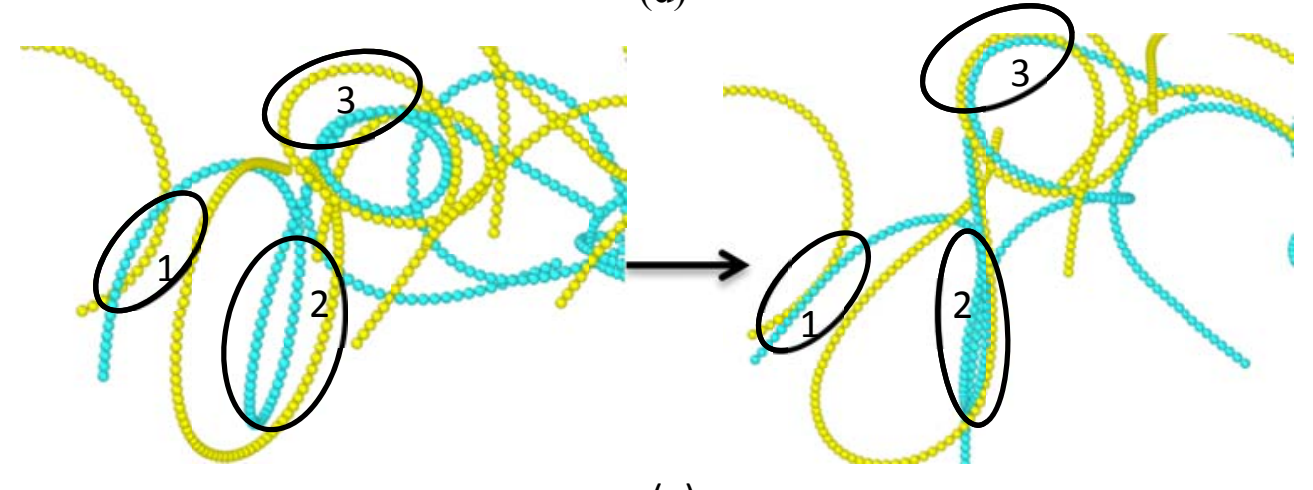

(e)

Figure 1: Computational cell of buckypaper for impact simulations and snapshots during the equilibrium process: (a) The initial configuration of random walk. (b) Side view of the relaxed model after equilibrium process. (c) Computation cell of buckypaper with receiver and impactor. (d) and (e) Zipping and attachment behavior of some typical SWCNTs extracted from the whole model during the equilibrium process. 

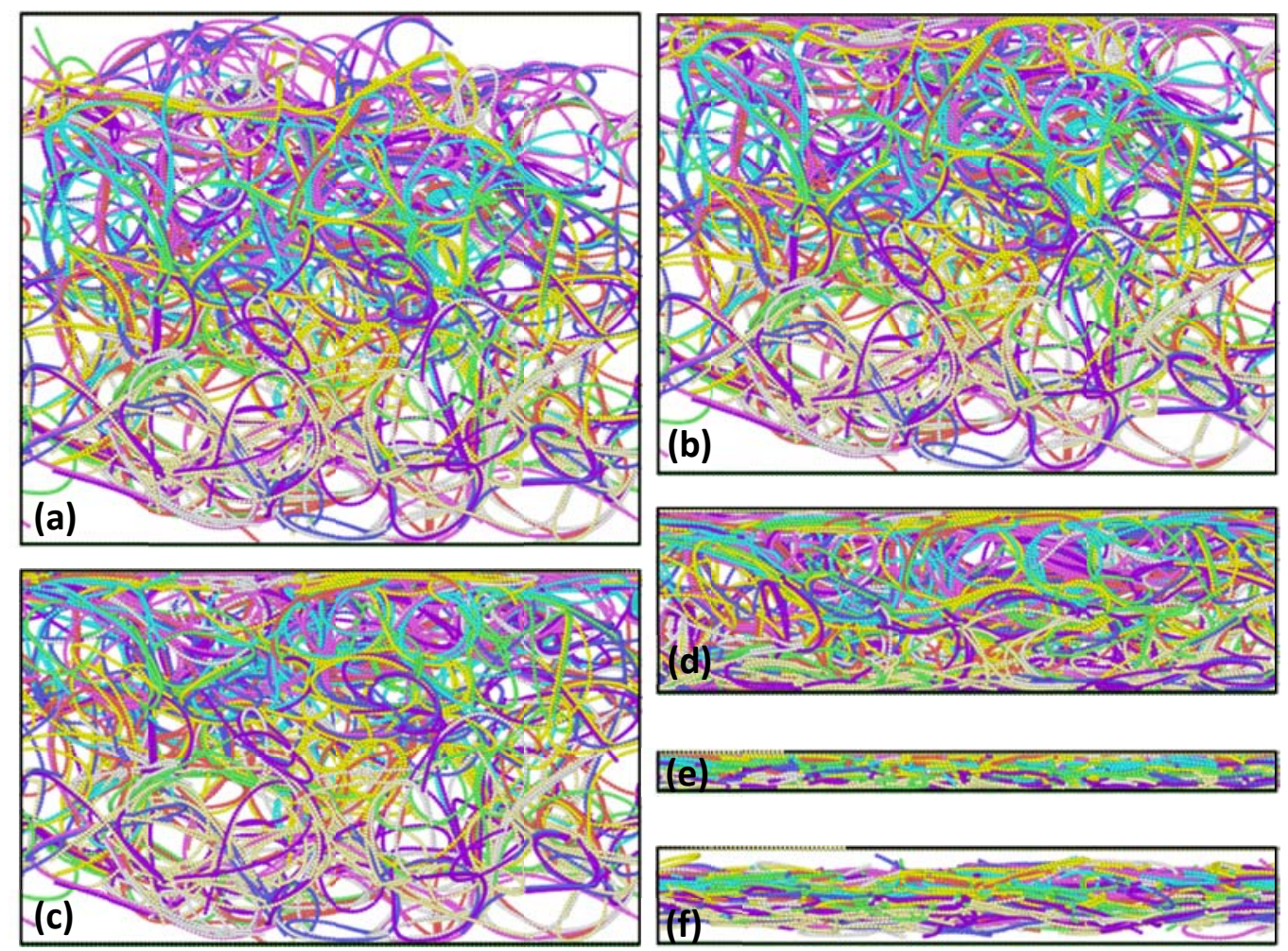

Figure 2: Deformation evolution of the buckypaper with density of $\mathbf{7 8 . 9 7} \mathbf{~ k g} / \mathbf{m}^{3}$, SWCNT length of $100 \mathrm{~nm}$, impact velocity of $200 \mathrm{~m} / \mathrm{s}$, and impact energy of $15 \mathrm{~J}$. (a) Initial configuration of the buckypaper. (b) Initial narrow densification region. (c) Straining arrives at the receiver. (d) Straining is transferred to the whole buckypaper. (e) The maximum compression distance. (f) Rebound of the impactor. 


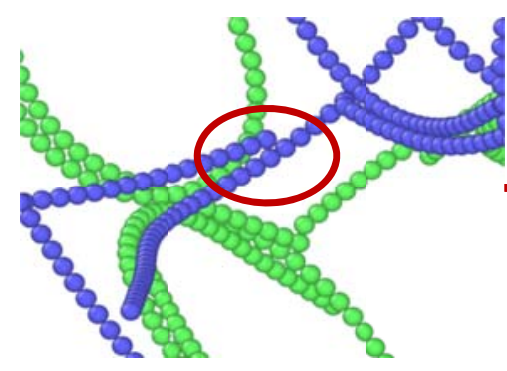

(a)
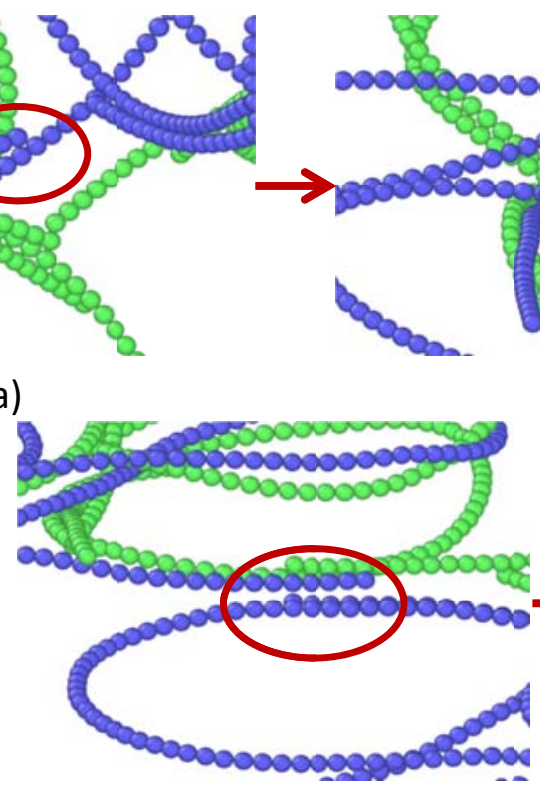

(d)

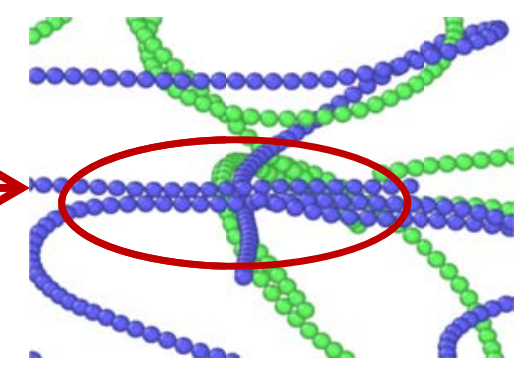

(c)

(b)

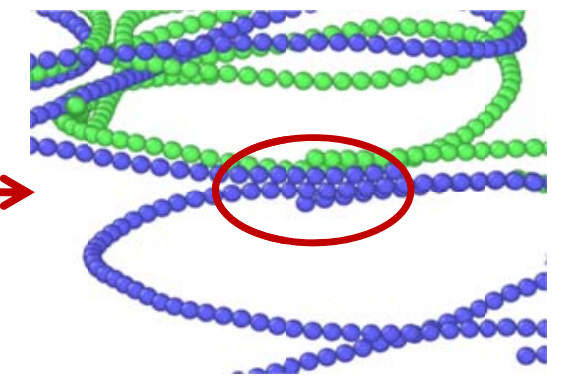

(e)

Figure 3: Detachment-attachment behavior of several selected SWCNTs from the $(5,5)$ SWCNT buckypaper during the impact process, while other SWCNTs remain hidden. (a) The original state of the buckypaper. (b) Detachment. (c)New attachment. (d)Detachment. (e)Attachment again. (d)-(e) happens recurrently. 

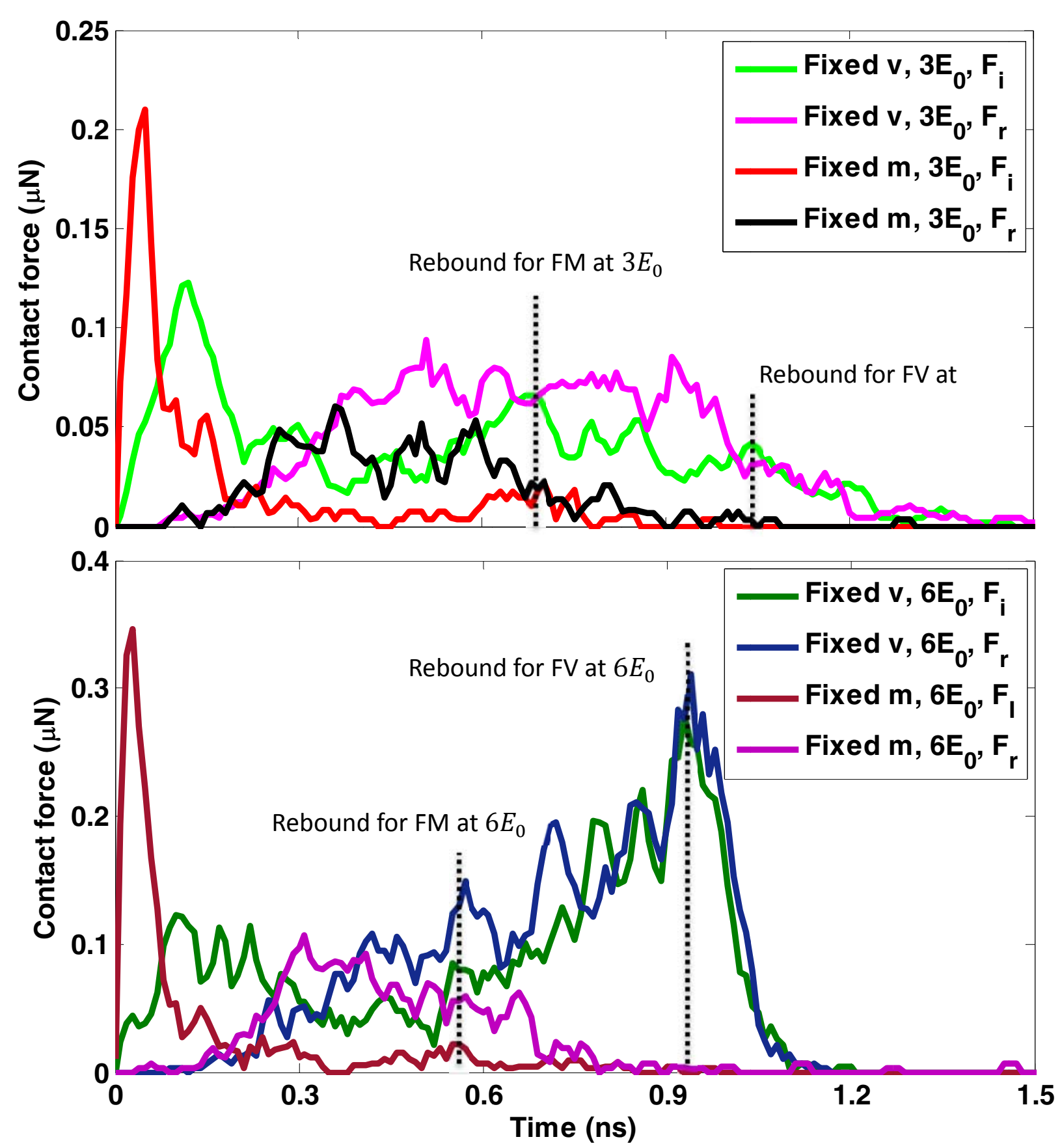

Figure 4: Contact forces between the impactor and buckypaper $\left(\boldsymbol{F}_{\boldsymbol{i}}\right)$ and between the buckypaper and the receiver $\left(\boldsymbol{F}_{r}\right)$ versus time under impact energy $\mathbf{3} \boldsymbol{E}_{\mathbf{0}}$ and $\mathbf{6} \boldsymbol{E}_{\mathbf{0}}$ for FV and FM cases, respectively. 


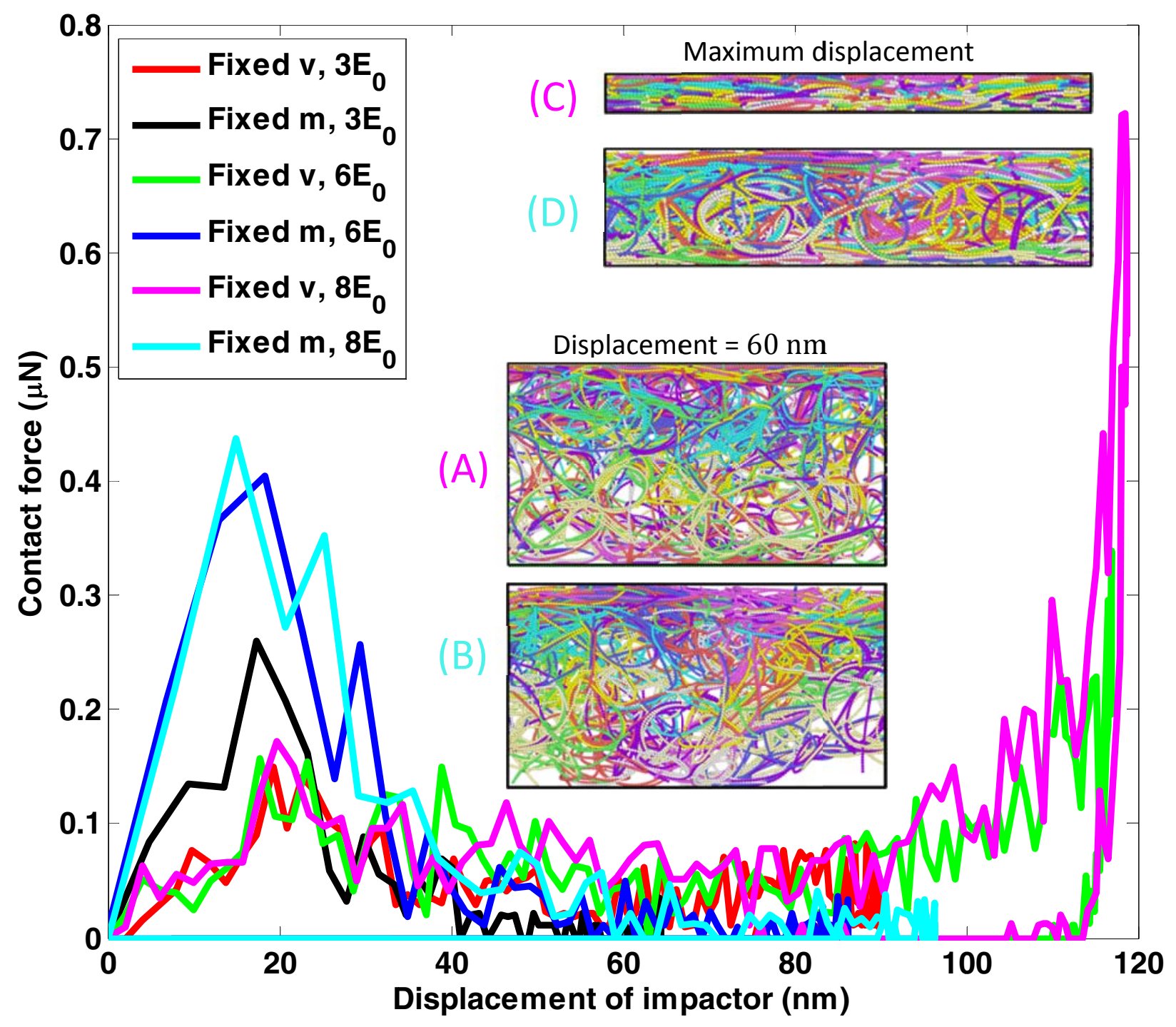

Figure 5: Contact force between the impactor and buckypaper versus displacement of the impactor at $3 \boldsymbol{E}_{\mathbf{0}}, \mathbf{6} \boldsymbol{E}_{\mathbf{0}}$, and $\mathbf{8} \boldsymbol{E}_{\mathbf{0}}$ for $\mathrm{FV}$ and $\mathrm{FM}$ cases respectively. Inserts show the morphologies under impact energy $8 \boldsymbol{E}_{\mathbf{0}}$ at the displacement of $\mathbf{6 0} \mathbf{n m}$ and maximum displacement respectively. The labels of the inserts have the same color as the corresponding curves. 


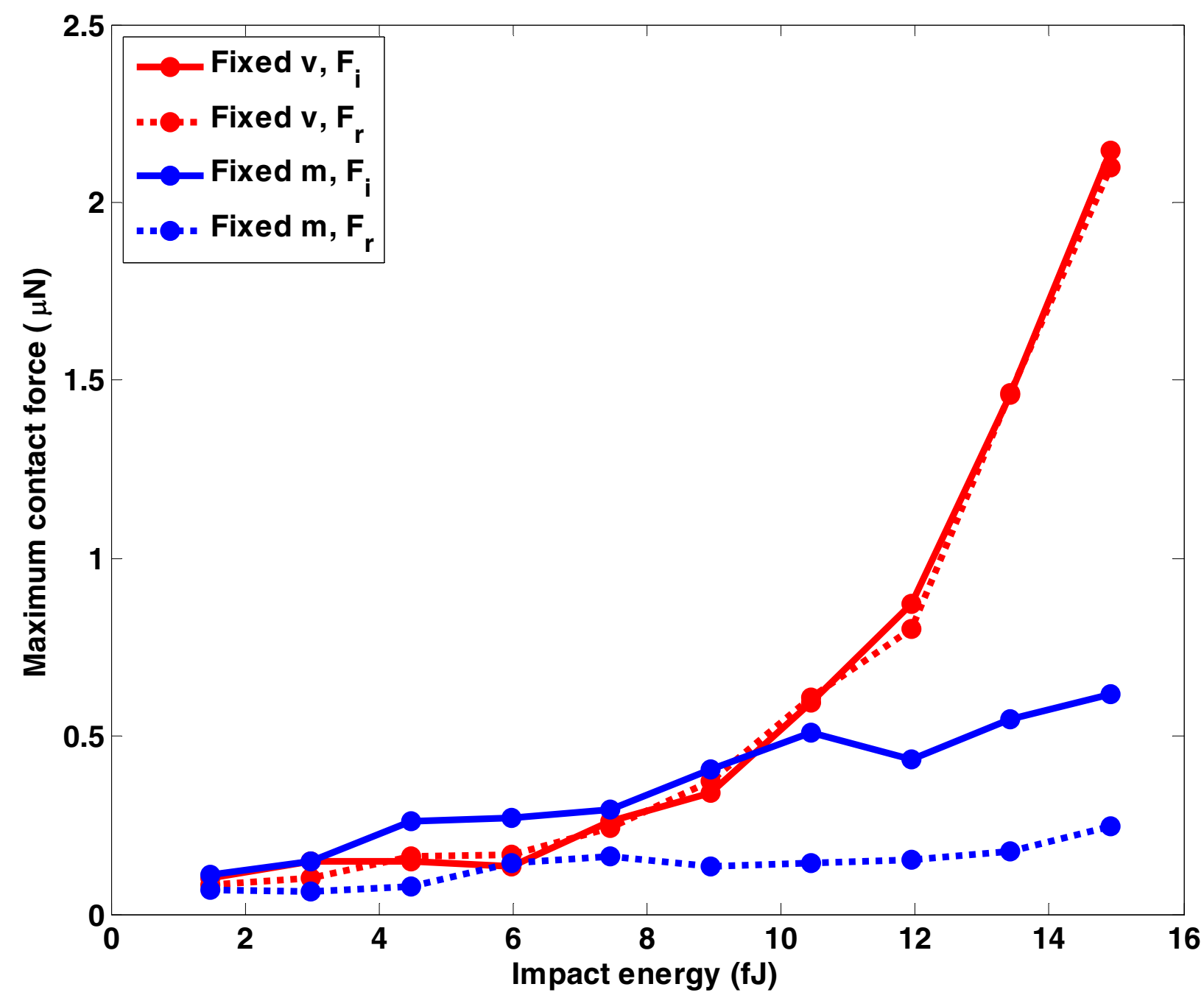

Figure 6: Maximum upper and lower contact forces versus impact energy for both FV and FM cases. 


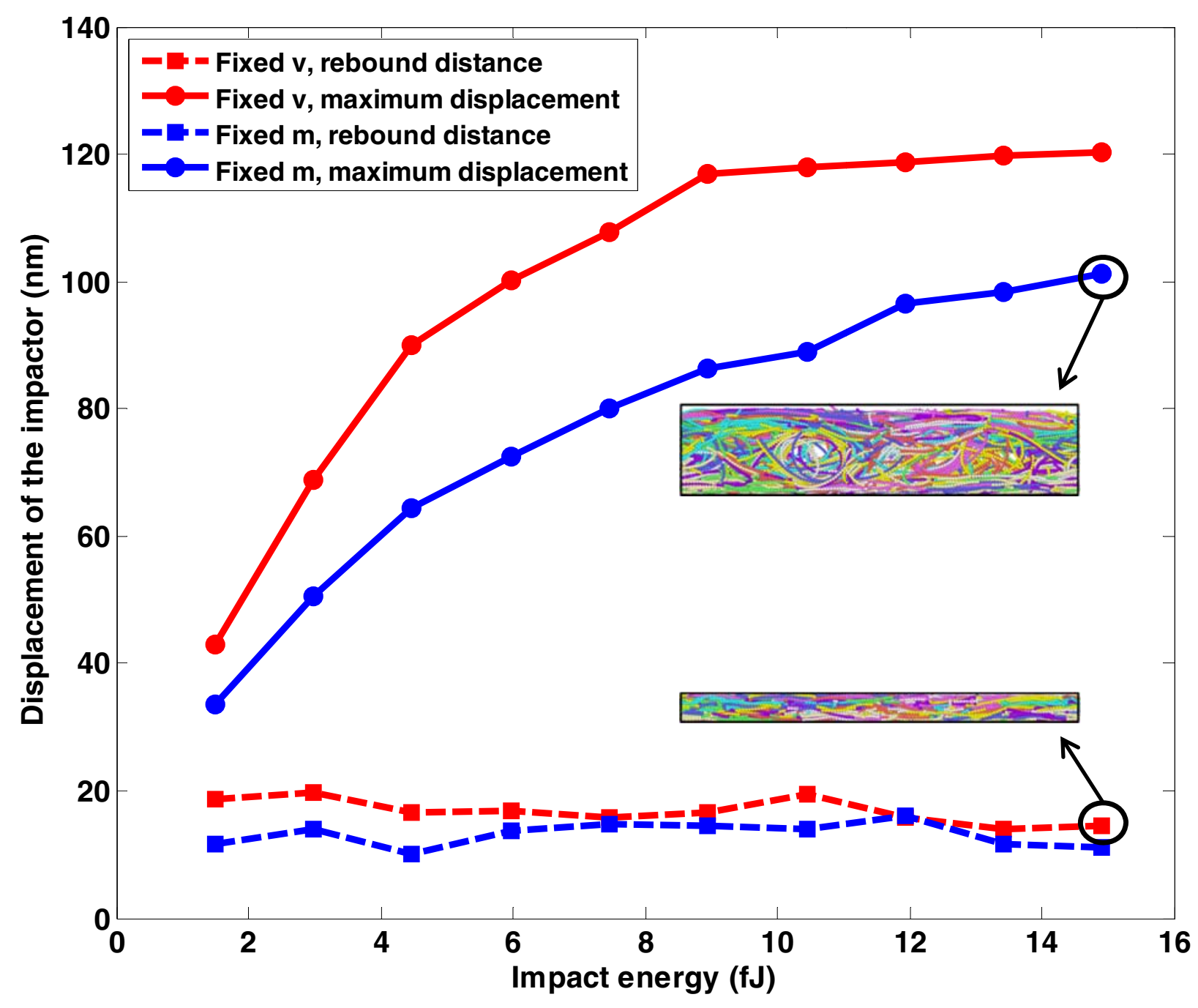

Figure 7: Maximum displacement of the impactor and rebound distance versus impact energy for FV and FM cases. Inserts show the morphologies of the buckypaper at the maximum compression distance. 


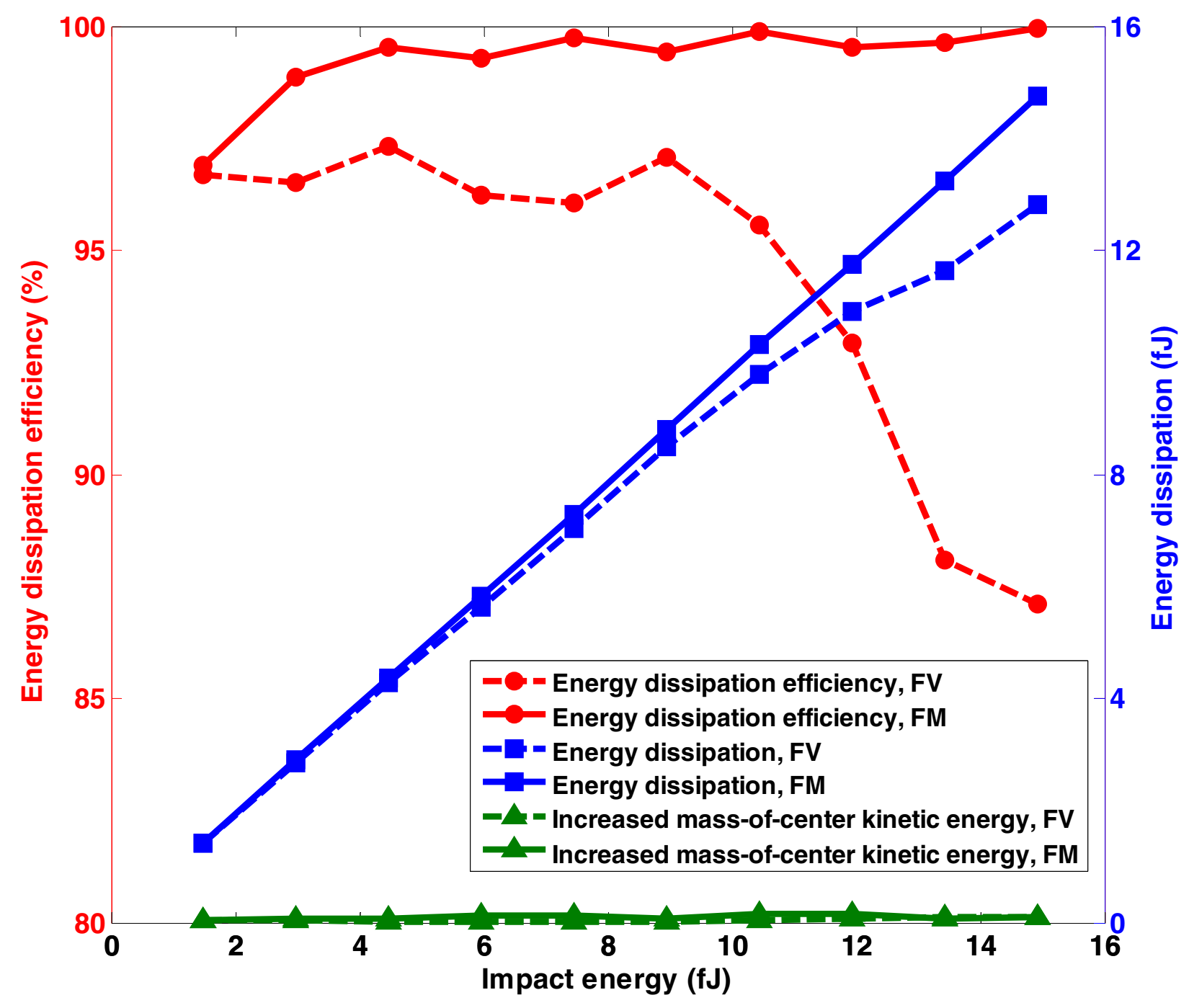

Figure 8: Energy dissipation efficiency and energy dissipation versus impact energy for FV and FM cases. 


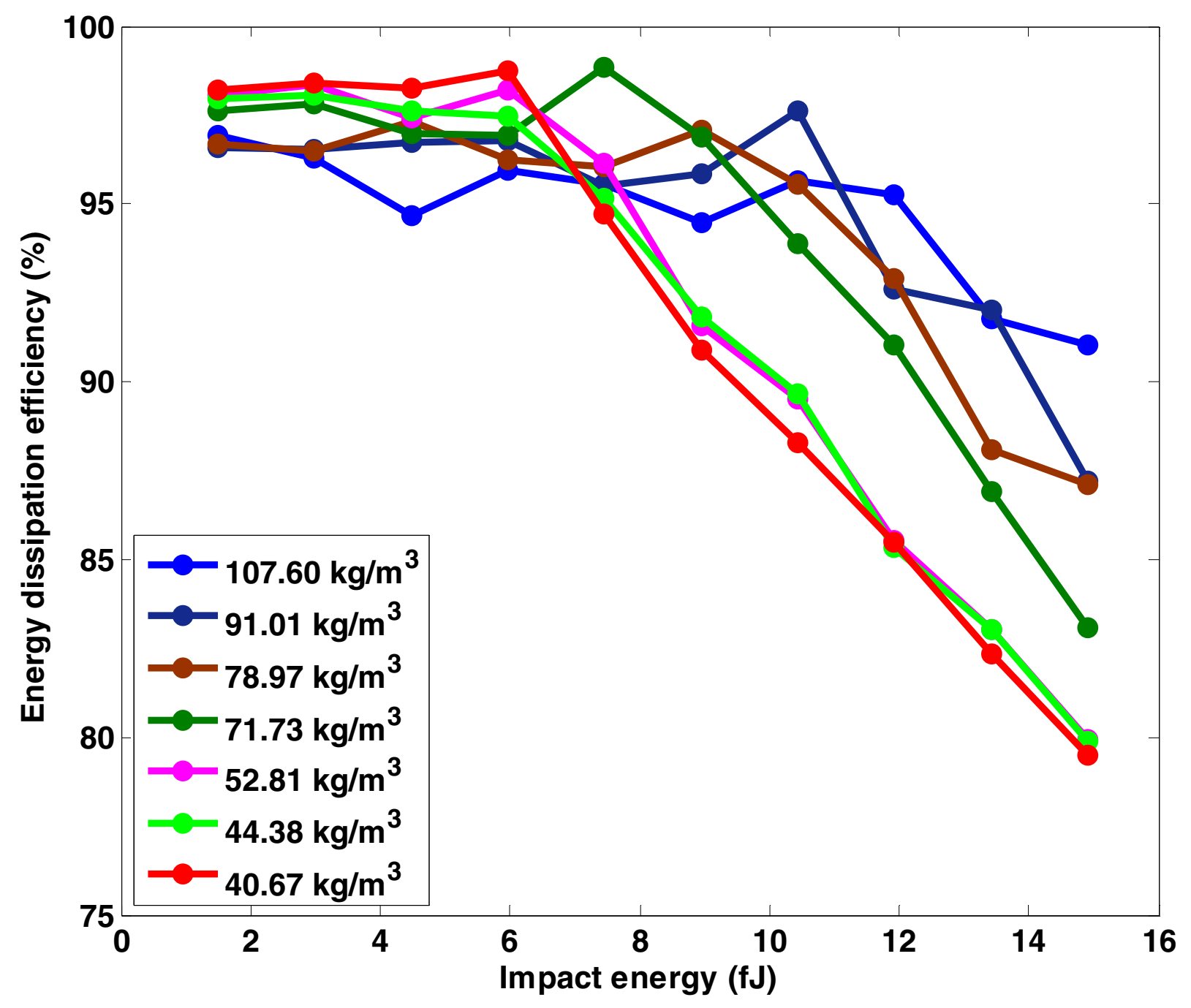

Figure 9: Energy dissipation efficiency of buckypapers with different densities versus impact energy. 

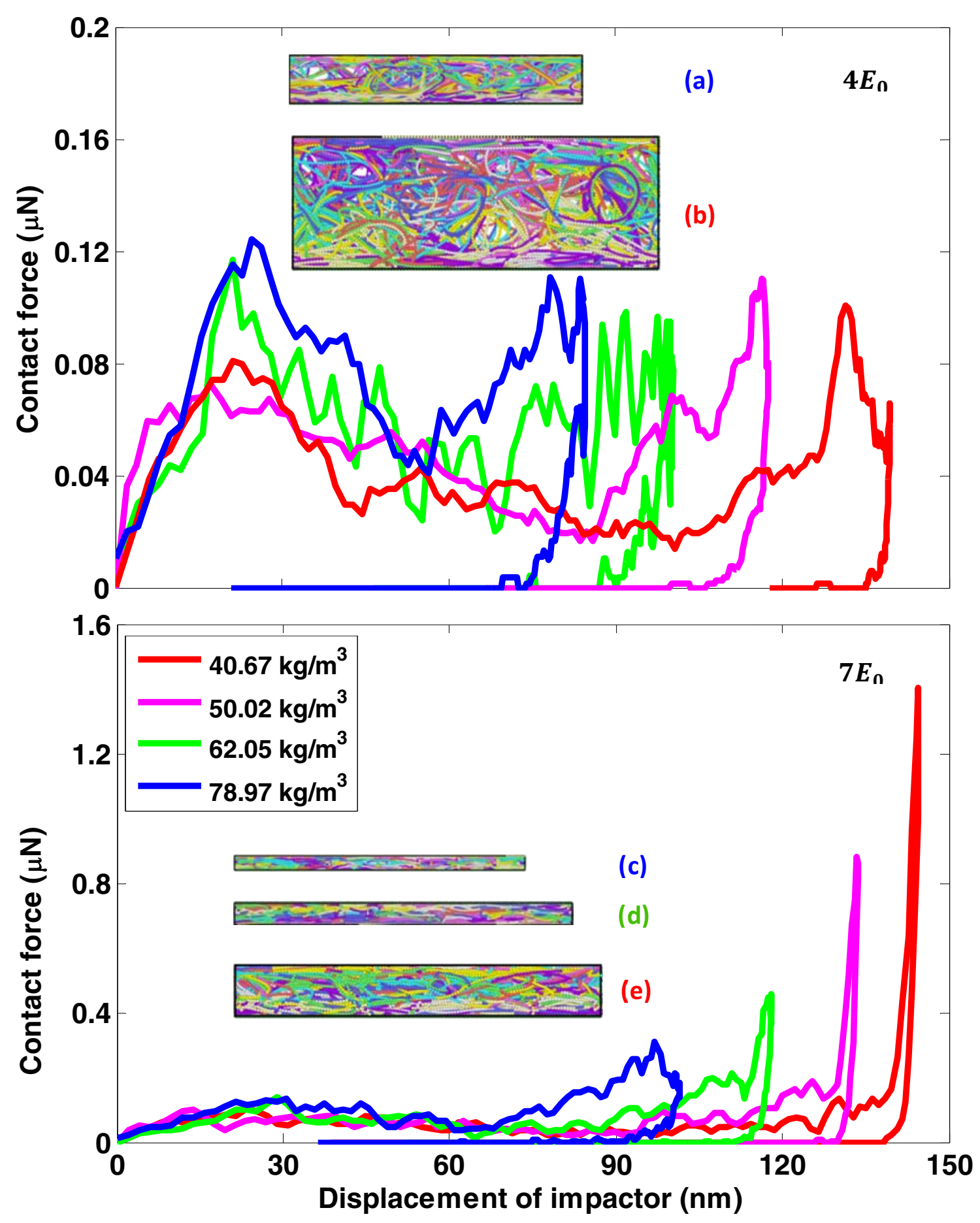

Figure 10: The upper contact force versus displacement of the impactor in the case of buckypapers with different densities. Inserts show the morphologies at the maximum compression distance. The labels of the inserts have the same color as the corresponding curves. 


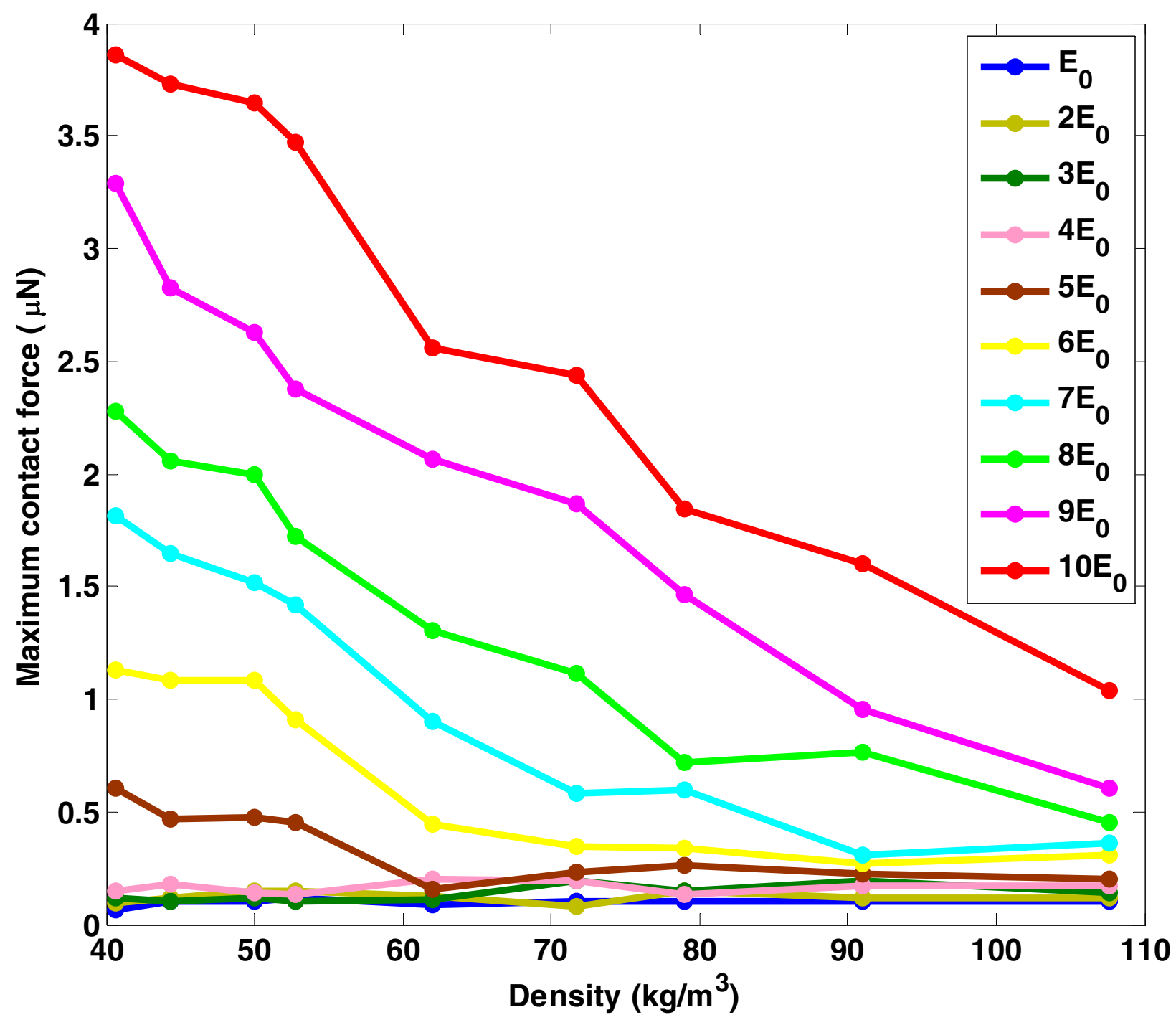

Figure 11: Maximum upper contact force versus density under impact energies varying from $\boldsymbol{E}_{\mathbf{0}}$ to $10 E_{0}$. 


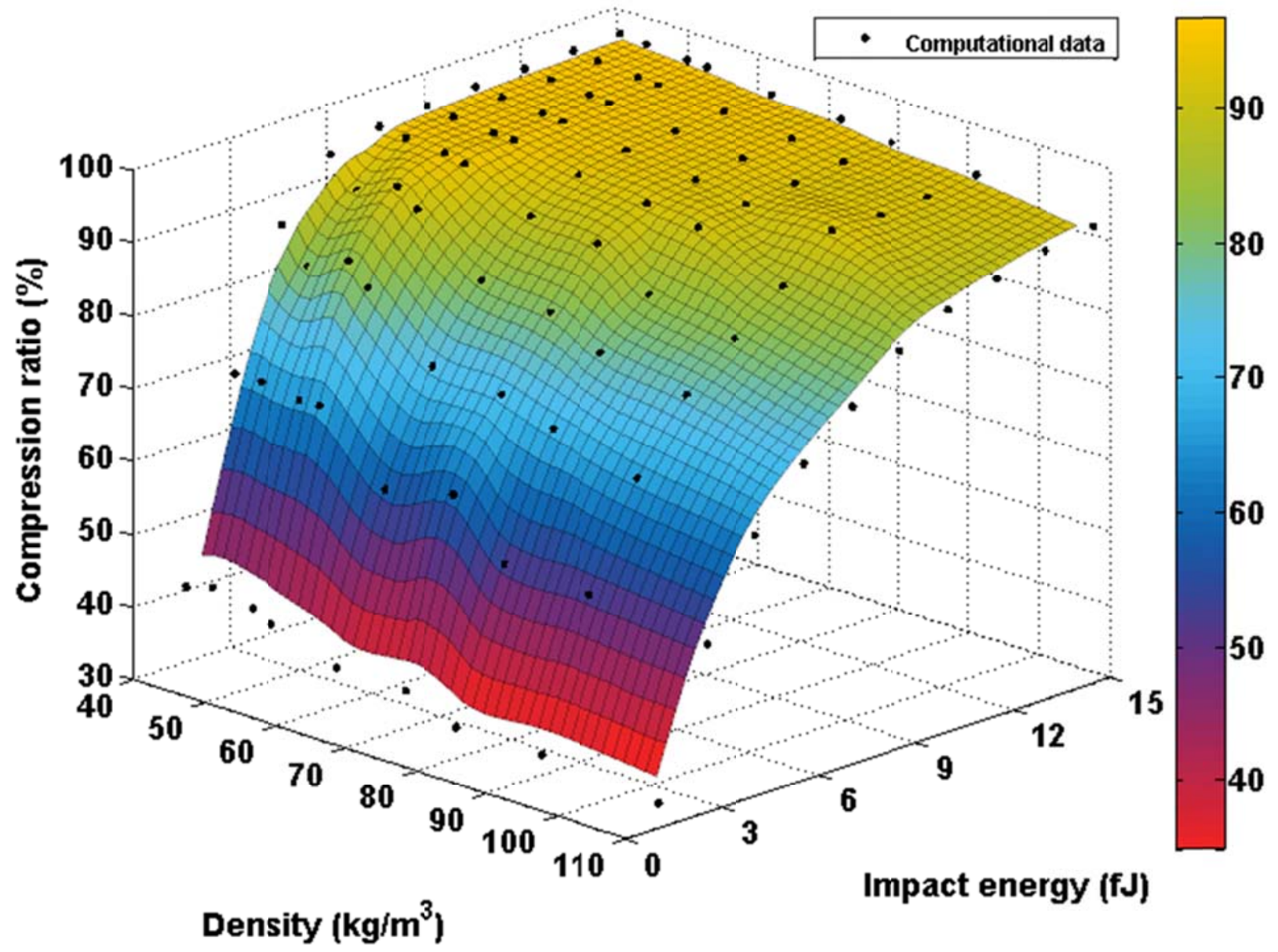

Figure 12: A fitting surface of the compression ratio as a function of the density of the buckypaper and the impact energy based on cubic spline interpolation. df 


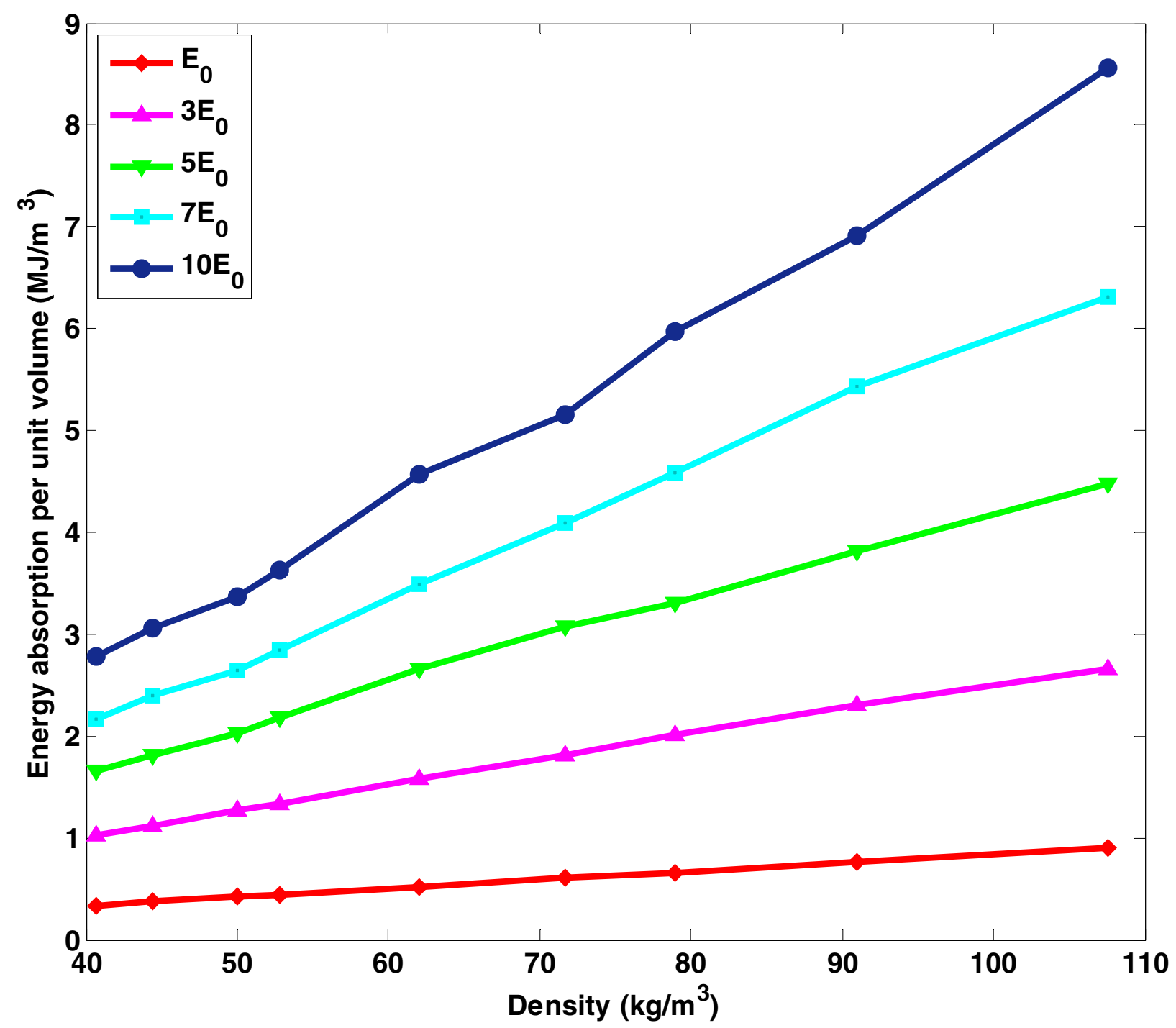

Figure 13: Energy absorption per unit volume of the buckypaper versus density under different impact energies. 


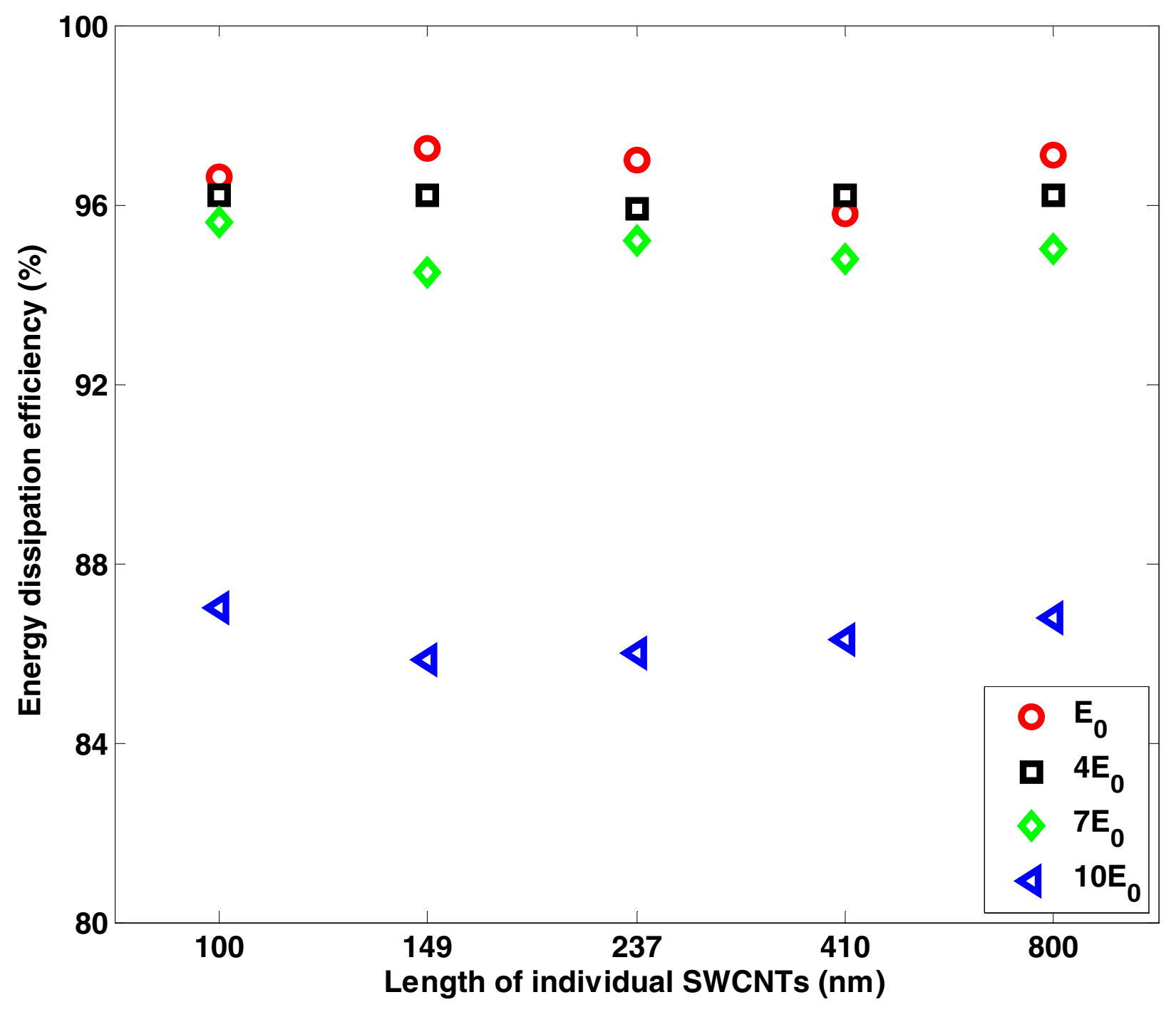

Figure 14: Energy dissipation efficiency of buckypaper with different lengths of individual SWCNTs under different impact energies. 


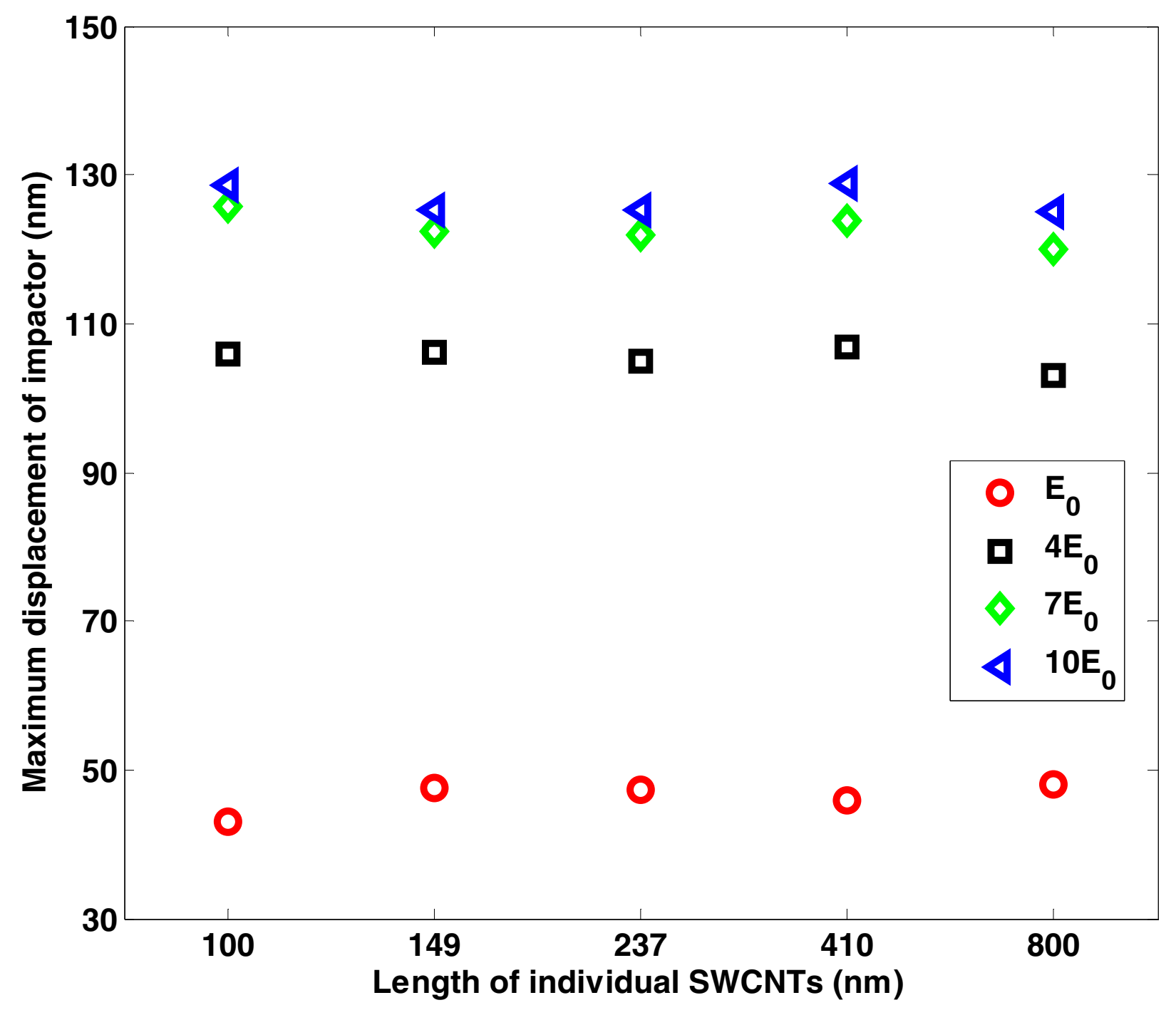

Figure 15: Maximum displacement of the impactor versus length for individual SWCNTs under different impact energies. 


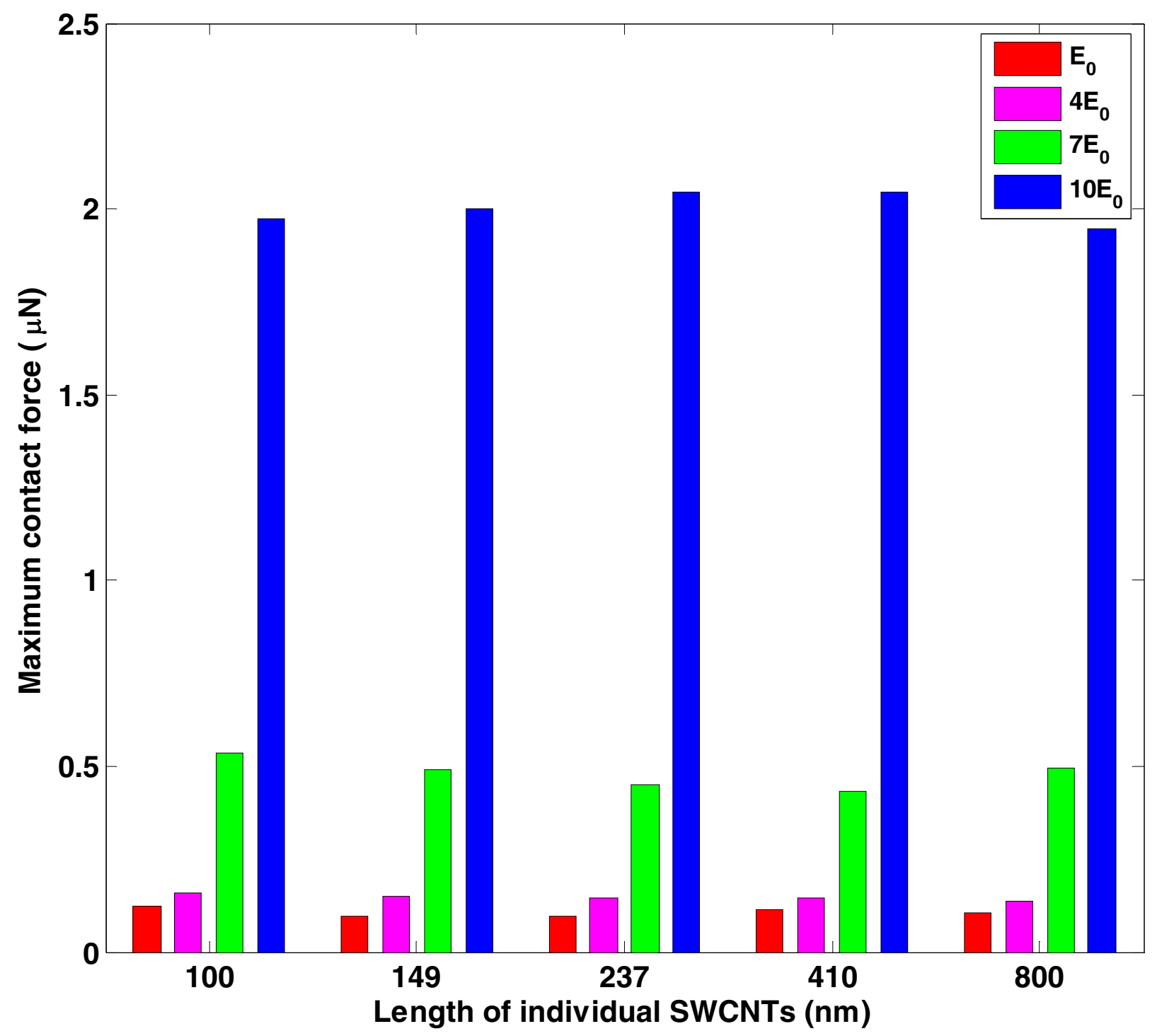

Figure 16: Maximum upper contact force versus length for individual SWCNTs under different impact energies. 